Division of Geological \& Geophysical Surveys

RAW-DATA FILE 2002-1

\title{
MAJOR OXIDE, MINOR OXIDE, TRACE ELEMENT, RARE-EARTH ELEMENT, TRACE GEOCHEMICAL, AND COAL QUALITY DATA FROM ROCKS COLLECTED IN EAGLE AND TANACROSS QUADRANGLES, ALASKA \\ IN 1999, 2000, and 2001
}

by

D.J. Szumigala, M.B. Werdon, R.J. Newberry, J.E. Athey, K.H. Clautice,

R.L. Flynn, J.C. Grady, W.C. Munly, and M.R. Johnson

$\$ 17.00$

June 2002

\section{THIS DATA COMPILATION HAS NOT BEEN REVIEWED FOR TECHNICAL CONTENT OR FOR CONFORMITY TO \\ THE PUBLICATION STANDARDS OF DGGS}

Released by

STATE OF ALASKA

DEPARTMENT OF NATURAL RESOURCES

Division of Geological \& Geophysical Surveys

794 University Avenue, Suite 200

Fairbanks, Alaska 99709-3645 


\section{CONTENTS}

PAGE

INTRODUCTION

1

ANALYTICAL METHODS

1

\section{TABLES}

TABLE 1. LOCATION AND DESCRIPTION OF ROCK SAMPLES COLLECTED IN THE EAGLE AND TANACROSS QUADRANGLES FOR TRACE ELEMENT GEOCHEMICAL ANALYSES.

TABLE 2. CONCENTRATION OF TRACE ELEMENTS IN ROCK SAMPLES COLLECTED IN THE EAGLE AND TANACROSS QUADRANGLES

TABLE 3. LOCATION AND DESCRIPTION OF ROCK SAMPLES COLLECTED IN THE EAGLE AND TANACROSS QUADRANGLES FOR MAJOR OXIDE, MINOR OXIDE, TRACE, AND RAREEARTH ELEMENT ANALYSES.

TABLE 4. CONCENTRATION OF MAJOR OXIDES, MINOR OXIDES, AND TRACE ELEMENTS IN ROCK SAMPLES COLLECTED IN THE EAGLE AND TANACROSS QUADRANGLES.

TABLE 5. CONCENTRATION OF RARE-EARTH ELEMENTS IN ROCK SAMPLES COLLECTED IN THE EAGLE AND TANACROSS QUADRANGLES

TABLE 6. PROXIMATE AND ULTIMATE COAL ANALYSES FROM ROCK SAMPLE COLLECTED IN THE EAGLE A-2 QUADRANGLE. 30

TABLE 7. DETECTION LIMITS FOR TRACE-ELEMENT GEOCHEMICAL ANALYSES .

TABLE 8. DETECTION LIMITS FOR MAJOR-OXIDE, MINOR-OXIDE, TRACE, AND RARE-EARTH ELEMENT ANALYSES

TABLE 9. DETECTION LIMITS FOR RARE-EARTH ELEMENT ANALYSES 33

\section{SHEETS}

SHEET 1. LOCATION MAP OF ROCK SAMPLES ANALYZED FOR TRACE ELEMENT GEOCHEMISTRY, AND MAJOR-OXIDES, MNOR OXIDES, TRACE ELEMENTS, AND (OR) RARE EARTH ELEMENTS, AND COAL QUALITY, EAGLE A-1, A-2, A-3, B-1, AND B-2 QUADRANGLES AND TANACROSS D-1 QUADRANGLE, ALASKA IN 1999, 2000, AND 2001, 1:63,360 SCALE, 1 SHEET (IN POCKET)

Note: This report (including all analytical data, tables, and map sheets) is available in digital format from the DGGS web site (http://wwwdggs.dnr.state.ak.us) at no charge. The digital data are available as PDF files and Excel spreadsheets. 


\title{
MAJOR OXIDE, MINOR OXIDE, TRACE ELEMENT, RARE-EARTH ELEMENT, TRACE GEOCHEMICAL AND COAL QUALITY DATA FROM ROCKS COLLECTED IN THE EAGLE AND TANACROSS QUADRANGLES, ALASKA IN 1999, 2000, and 2001
}

\author{
by \\ D.J. SZUMIGALA', M.B. WERDON', R.J. NEWBERRY², J.E. ATHEY', KH. CLAUTICE', R.L. FLYNN ${ }^{2}$, J.C. \\ GRADY', W.C. MUNLY', AND M.R. JOHNSON ${ }^{2}$
}

\section{INTRODUCTION}

Mineral resource personnel from the Alaska Division of Geoiogical \& Geophysical Surveys carried out a geological field survey, including mapping and sampling of Eagle and Tanacross quadrangles, Alaska from June 1999 to July 2001. The fieldwork provides basic information critical to building an understanding of Alaska's geology and is part of an integrated program of airborne geophysical surveys followed by geological mapping programs. During 2001, 65 rock samples were collected for geochemical trace-element analysis, and 145 samples were collected for whole rock (major and minor oxides, and petrogenetically important trace and (or) rare earth element data) analysis. This report also includes 18 rock samples collected for geochemical trace-element analysis, 59 rock samples collected for whole rock analysis, and $1 \mathrm{coal}$ analysis collected during 1999 and 2000 that were not previously released in DGGS reports (Raw-Data-File 2000-1 and Raw-Data-File 2000-4). The locations of all samples listed in the tables of the current report are shown on Sheet 1. Location data (in UTM coordinates with a Clark 1866. NAD27, UTM zone 7 projection), descriptions, and analytical results for each sample are tabulated in tables 1-6.

\section{ANALYTICAL METHODS}

Chemex Labs, Inc perforned all trace-element geochemical analyses in this report. Rock samples were crushed so that at least 70 percent of the material passed through a $-10(2 \mathrm{~mm})$ mesh screen. A 200-gram, representative split of the sample was then taken using a riffle splitter. The 200 -gram sample was then pulverized in a chrome steel ring mill so that 95 percent of the sample passed through a -150 (106 micron) mesh screen. DGGS also submitted geochemical standards at an approximate rate of one pulp per fifteen unknowns, and crushed granite and basalt standards were submined at an approximate rate of one whole rock standard per fifteen unknowns.

Gold was analyzed on a 30-gram representative sample split using Fire Assay (FA) and Atomic Absorption Spectroscopy (AAS) methods. Most trace elements were analyzed by the Inductively Coupled Plasma - Atomic Emission Spectroscopy (ICP-AES) methods after nitric aqua regia digestion. Platinum and palladium were analyzed by the Fire Assay - Inductively Coupled Plasma (FA-ICP) method. Analytical detection limits are tabulated for the geochemical analyses in table 7.

Chemex Labs, Inc performed all whole rock analyses in this repon Major and minor element oxides were determined by XRF methods following a metaborate fusion. Trace elements ( $\mathrm{Ba}, \mathrm{Nb}, \mathrm{Rb}, \mathrm{Sr}, \mathrm{Y}$, and $\mathrm{Zr}$ ) were also analyzed using XRF methods on a pressed pellet. Rare Earth elements were analyzed using Inductively Coupled Plasma - Mass spectroscopy after fusing with lithium metaborate. Analytical detection limits for the major-oxide, minor-oxide, trace, and rare-earth element analyses (XRF method) are tabulated in table 8.

The coal sample was analyzed by Geochemical Testing, a division of Energy Center, Inc. The sample submitted to the lab weighed approximately five pounds and represented a composite of several coal layers within Tertiary sedimentary rocks. For practical purposes, the chemical composition of coal is given in terms of its "proximate" analysis and by its "ultimate" or elemental composition. Proximate analysis decermines moisture content, volatile marter content (V.M.), ash and, indirectiy, the fixed carbon content (F.C.) which is expressed as a percentage of the dry coal mass and defined by: $\% F . C .=100-(\%$ V.M. $+\%$ ash $) \quad$ Ultumate or elemental analysis involves a quantitative determination of the percentage of carbon, hydrogen, nitrogen and organic sulfur with oxygen usually determined by difference. Specific energy determination determines the heat energy produced from the combustion of each gram of coal. Neither proximate nor ultimate analysis gives much information about coal structure but both give information that, in light of experience can be directly correlated with the behavior of coal in most industrial processes. Results are given in table 6 . Analytical detection limits for the rare-earth element analyses determined by ICP-MS are shown in table 9.

\footnotetext{
Affiliations

' Alaska Division of Geological \& Geophysical Surveys

${ }^{2}$ University of Alaska Fairbanks
} 
Table 1. Location and description of rock samples collected in the Eagle and Tanacross quadrangles for metal and trace element geochemical analyses.

\begin{tabular}{|c|c|c|c|c|c|}
\hline SAMPLE & UTME & UTMN & \multicolumn{2}{|c|}{ Quadrangle } & Description \\
\hline ŌORF́233C & 494403 & 7107903 & Eagle & A-1 & Vuggy, fine-grained quartz breccia with abundant iron-oxide stain \\
\hline OORF86.1B & 492519 & 7096775 & Tanacross & D-1 & Dark gray, coarse-grained biotite-homblende gneiss with disseminated pyrite \\
\hline OOORF187C & 496803 & 7099341 & Eagle ${ }^{-}$ & A-1 & Bluish purple to purplish black magnetite-serpentine rock with magnetite layers up to $4 \mathrm{~mm}$ wide. \\
\hline $00 R F 2 \overline{1} \bar{B}$ & $495 \overline{3} 34$ & $71016 \overline{3} 7$ & Eagle & $\mathrm{A}-1$ & Bright green, fine-grained serpentinized ultramafic with iron oxide stain.. \\
\hline $00 \bar{R} \bar{F} 22 \overline{2} A$ & $49 \overline{4} \overline{6} \overline{2} \overline{4}$ & $7101 \overline{9} \overline{3}$ & Eagle & $\overline{\mathrm{A}}-\mathrm{j}$ & Plagioclase hornblendite with equant homblende up to $1 \mathrm{~cm}$, iron oxide and sparse calcite veinlets \\
\hline F246B & 494913 & 7100453 & Eagle & A-1 & Select sample of dark gray chromitite with submetallic luster \pm iron oxide, $>95 \%$ chromite up to $>1 \mathrm{~cm}$. \\
\hline \&F333 & 497580 & 7103347 & Eagle & $\dot{\mathrm{A}}-1$ & Carbonaceous-white mica-quartz schist and quartzile with abundant disseminated pyrite and iron oxide. \\
\hline OORF405B & 496583 & 7108461 & Eagle & A-1 & Select sample of gray, fine grained quartz-calcite vein with green specks, sparse pyrite and abundant iron oxide. \\
\hline $0 \bar{R} F 422$ & $4 \overline{9} 4 \overline{5} \overline{9}$ & 7094963 & Tanacross & D- 1 & Select sample of light gray, quartz-calcite vein with iron-oxides and minor disseminated molybdenum flakes. \\
\hline $00 R F 463 \mathrm{~A}$ & 496200 & 7094453 & Tanacross & D-1 & Very coarse grained, unfoliated biotite hornblendite with iron oxide staining. \\
\hline 00RF463C & 496200 & 7094453 & Tanacross & D-1 & Dark gray, very coarse-grained, unfoliated hornblendite with abundant disseminated pyrite and iron oxide. \\
\hline $0 ̈ 0 R F 484 B$ & $49 \ddot{7} 7177$ & 7097183 & Eağle & A-1 & Dark gray, carbonaceous-biotite-quart schist with disseminated pyrite up to $5 \mathrm{~mm}$ diameter. \\
\hline ÖORF490A & 498781 & $70975 \overline{83}$ & Eagle & A-1 & Carbonaceous white mica-quartz schist, and quartz breccia with very abundant iron oxide. \\
\hline OORF507B & 498980 & 7098640 & Eagle & A-1 & Select sample of quartz breccia with very abundant iron oxide. \\
\hline OORF510 & 492405 & 7097039 & Eagle & A-1 & Medium to coarse grained marble \pm fuchsite with sparse disseminated pyrite and iron oxide after pyrite \\
\hline 5208 & 499583 & 7094051 & Tanacross & D-1 & Very coarse-grained, tourmaline granite orthogneiss; some muscovite and abundant tourmaline up to $2 \mathrm{~cm}$ long. \\
\hline OORF552 & 499764 & 7107719 & Eagle & A-1 & Medium-grained, chlorite-while mica-quartz schist with iron oxide after pyrite up to $1 \mathrm{~cm}$ diameter along foliation. \\
\hline OORF607 & 497665 & 7105470 & Eagle & A-1 & Dark gray, carbonaceous-white mica-quartz-fuchsite schist with abundant iron oxide after pyrite along foliation \\
\hline 01JEAं56A & 484866 & 7109533 & Eagle & A1 & Grab sample of biotite-quartz-feldspar gneiss with pyrite-bearing pegmatite or quartz vein. \\
\hline $01 \mathrm{JEA} 207 \mathrm{~A}$ & 490960 & 7112584 & Eagle & A1 & Select sample of highly iron-oxide stained breccia?, fault gouge or gossanous material with blac \\
\hline 01 JEA219A & 493707 & 7122483 & Eagle & $\mathrm{Ai}$ & Select sample of highly iron-oxide stained, quartz-(carbonate) vein with occasional fine-grained gray metal specks. \\
\hline 01JEA219E & 493707 & 7122483 & Eagle & A1 & Grab sample of fractured quartz veins in gneiss or silicified gneiss with 5 percent fine-grained pyrite. \\
\hline EA373A & 496068 & 7111925 & Eagle & A1 & Grab sample of biotite-quartz-feldspar gneiss with trace pyrite on fracture. \\
\hline $01 \mathrm{KCO} 1 \mathrm{~B}$ & 479905 & 7098854 & Eagle & $\mathrm{Ai}$ & Select sample of a 8 inch thick pod of leucocratic gneiss with minor disseminated pyrite, possibly other ?sulfides? \\
\hline $01 \mathrm{KC6B}$ & 477939 & 7101406 & Eagle & A1 & Select sample of most iron-oxide slained quartz boudined, calcareous chlorite-muscovite-feldspar-quartz gneiss. \\
\hline $\mathrm{C} 30 \mathrm{~B}$ & 488250 & 7100300 & Eagle & A1 & Select sample of iron-oxide stained garnet-chlorite-quart-(feldspar?) gneiss with pyrite near 1 foot-wide quartz vein. \\
\hline $01 \mathrm{KC} 43 \mathrm{~A}$ & 485839 & 7110309 & Eagle & A1 & rab sample of aplitic pegmatite with occasional orthogneiss. \\
\hline Č́5̈̈B & $4 \overline{8} \overline{8} \dot{9} \overline{7} \overline{8}$ & $711886 \overline{1}$ & Eagle & A1 & Select sample of light orange-colored portion of 4 foot diameter white quartz boulder \\
\hline $01 \mathrm{~K} \overline{\mathrm{C}} 5 \mathrm{5} \mathrm{C}$ & 489527 & 7118869 & Éggle & $\bar{A} \overline{1}$ & Select sample of quartz + chlorite vein. Fair amount of quart veining in the area. Follow this one for $20 \mathrm{ft}$. \\
\hline $01 \mathrm{KC59B}$ & 494088 & 7113359 & Eagle & A1 & Select sample of one-inch thick pegmatite dike. \\
\hline & 494073 & 7113541 & Eagle & Á1 & Anastamosing pegmatite dikes in biotite granodiorite ranging from 1 to 8 inches thick. \\
\hline$\overline{01 \mathrm{~K}} \overline{\mathrm{C}} \overline{\mathrm{6}} \overline{1} \overline{\mathrm{B}}$ & $4 \overline{9} 4085$ & 7113993 & Eagle & A1 & Select sample of pyritic quartz veins that crosscut foliation of biotite granodiorite. \\
\hline $01 \mathrm{KC6} 2 \mathrm{~A}$ & 493982 & 7113977 & Eagle & A1 & rab sample of anastomosing pematitic veins that range up to several feet in thickness. \\
\hline $01 \mathrm{KC66 \textrm {A }}$ & 494842 & 7110688 & Eagle & A1 & Select sample of finely laminated quartite with some quartz veins and finely disseminated pyrite along joints. \\
\hline $01 \mathrm{KC} 6 \overline{6} \mathrm{~B}$ & $49484 \overline{2}$ & $71106 \overline{8}$ & Eagle & A1 & Medium- to coarse-grained amphibolite in contact with thinly laminated quartzite. \\
\hline & 494581 & 7111072 & Ëäle & A1 & Gray quankite with minor brecciation and some quartz veining, also slickensides and very jointed. \\
\hline & 494095 & $711290 \overline{5}$ & Eagie & A1 & Pyritic, foliated hornblende-biotite granodiorite dike crossculting foliation of marble. \\
\hline & 494137 & $\overline{7} 113663$ & Eagle & A1 & Aplitic pegmatite with trace pyrite. \\
\hline $01 \mathrm{KC76B}$ & 491610 & 7112774 & Eagle & A1 & Select sample of quartz-carbonate veins from 1 to $5 \mathrm{~cm}$ wide in quartz-rich gneiss. \\
\hline $01 \mathrm{KC77B}$ & 491738 & 7113074 & Eagle & A1 & Select sample of quartz-carbonate veins from 1 to $5 \mathrm{~cm}$ wide in amphibolite. \\
\hline $01 \mathrm{KC} \overline{\mathrm{B}} \overline{\mathrm{A}} \mathrm{A}$ & $4 \overline{9} \overline{27} \overline{7} \overline{7}$ & $\overline{7} 12 \overline{4} 4 \overline{8} \dot{5}$ & Ëaglè & A1 1 & Select sample of 10-inch layer of pink marbie within white marble. Possible small specks around silvery bleb. \\
\hline
\end{tabular}


Table 1. Location and description of rock sampies collected in the Eagle and Tanacross quadrangles for metal and trace element geochemical analyses.

\begin{tabular}{|c|c|c|c|c|c|}
\hline SAMPLE & UTME & UTMN & \multicolumn{2}{|c|}{ Quadrangile } & Description \\
\hline $01 \mathrm{KC} 92 \mathrm{~A}$ & 493212 & 7124832 & Eagle & Bi & Select sample of gossanous, sheared, schistose gneiss with quartz veins containing arsenopyrite or stibnite. \\
\hline $01 M B W 19 \mathrm{~A}$ & 483460 & $709912 \pi$ & Eaglè & Aí & Marble with nearby vuggy quartz-carbonate breccia and quartz veins up to $1 \mathrm{~cm}$ wide cutting quartz in breccia. \\
\hline 01MBW39c & 491333 & 7100713 & Eagle & At & Orange-colored, limonite stained white and tan layered quartzite and weathered feidspar. \\
\hline OIMBW41A & 491446 & 7100801 & Eagle & A1 & Select sample of quartz-pyrite veins up to 2 inches wide cutting argilfically altered and brecciated gneiss. \\
\hline 01MBW45A & 491587 & 7101353 & Eagle & At & Select sample of brecciated and vuggy quartz vein with fine-grained pyrite. \\
\hline 01MBW79A & 484774 & 7103678 & Eagle & A1 & Highly weathered and slightly porous/leached green and red skarnoid? with clear gray quartz stringers. \\
\hline 01MBW80B & 484655 & 7103682 & Eagie & Ai & Gray, foliated quartzite with $3 \mathrm{~mm}$ thick marble layers and mm-thick layers of actinolite-chlorite-pyrite-limonite \\
\hline $01 \mathrm{MBW} 143 \mathrm{~A}$ & 488002 & $711325 j$ & Eagle & At & Gossaneous, limonite stained, brecciated rock with open fractures and quartz veining. \\
\hline Ö1MBW'146A & 488100 & $\overline{7} 1128 \overline{7} \overline{7}$ & Éagle & Ai & Limonite-stained, partially gossaneous, Teached high-grade metamorphic rock cut by thin quartz vein. \\
\hline O1MBWI56A & 489419 & $71147 \overline{9}$ & Eagle & $A 1$ & Probable fault zone consisting of milky quartz vein and altered rock breccia, with limonite-filed vugs. \\
\hline 01MBW158A & 489474 & 7114735 & Eagle & A1 & Limonite-stained, leached, sericite-altered micaceous quartzite or schist with 1-2 mm diameter limonite spots. \\
\hline $01 \mathrm{MBW} 230 \mathrm{~B}$ & $4926 \overline{63}$ & $712 \overline{3} 981$ & Eagle & $\mathrm{A} \uparrow$ & Sefect sample of $\uparrow$-foot wide milky quartz vein crosscutting foliation in biotite schist and gneiss. \\
\hline $01 \mathrm{MBW}$ & $4 \overline{9} \overline{3} \overline{\overline{6}} \overline{3}$ & $712 \overline{3} \overline{7} 0 \overline{5}$ & Eagle & A 1 & Fine- to medium-grained quartzite with limonile staining. \\
\hline $01 M B W 237 \mathrm{C}$ & 493486 & $\overline{7} 1 \overline{2} \overline{2} \overline{\overline{2}} \overline{2} 2$ & Eagle & Aj & Select sample of most limonile-stained and sericite-rich bioitite schist and biotite-quartz-feldspar gneiss. \\
\hline $01 M B W 248 A$ & 495875 & 7113318 & Eagle & $\mathrm{A}^{\mathrm{j}}$ & Fractured white mica bearing quarticite with limonite stain along fractures. \\
\hline$\overline{0} 1 \mathrm{MBBW}=21 \mathrm{~A}$ & $495725^{\circ}$ & 7113372 & Eagle & A1 & Limonite-stained rock, possibly dike or metadike with quartz in rock looking like foliation-parallel veins. \\
\hline $01 \mathrm{MBW} 280 \mathrm{~A}$ & 493500 & $7 \dot{105} \overline{\overline{8}} \dot{2}$ & Eagle & A1 & Limonite-stained, siliceous, gossaneous rock cut by numerous quartz microfractures. \\
\hline $01 \mathrm{MBW} 2 \mathrm{~B} \dot{8}$ & 492400 & 7105836 & Ëagle & Ai & Green, fine-grained, epidote-hornblende massive homfels with quartz-epidote-iron sulfide veins up to $1 \mathrm{~cm}$ wide. \\
\hline $\mathrm{B} W 28 \overline{4} \mathrm{C}$ & $492400^{\circ}$ & 7105836 & Ëaglè & A1 & Coarse-grained cfinopyroxenite with minor calcite and iron sulfides. \\
\hline $01 \mathrm{MBW} 3 \overline{3} 51 \overline{\mathrm{A}}$ & $4 \overline{9} \overline{4} 455$ & 7122255 & Eagle & $A i^{-}$ & Tan, hematite-stained and quartz-veined quartz-feldspart greiss \\
\hline $01 \mathrm{MBW} 362 \mathrm{~B}$ & $4866 \overline{9}$ & $\overline{709744 \overline{2}}$ & Ëagle & Ai 1 & 6 inch thick layer/band of orthogneiss containing 3 percent pyrite altered to limonite within paragneiss. \\
\hline $01 \mathrm{MBW} 3 \mathrm{~B} 5 \mathrm{~A}$ & $482951^{\circ}$ & $7 \overline{12} \overline{0} \overline{6} \overline{7}$ & Ëağle & $A \overline{1}$ & Coarsegrained amphibole-feldspar gness containing up to 3 percent pyrhotite in patches and microveinlets. \\
\hline$\overline{0} \mathrm{MB} \overline{\mathrm{W}} \overline{3} \overline{8} \overline{\mathrm{A}}$ & 482973 & $7 \overline{120839}$ & Eagle & A1 & C̄oarse-graned amphibole-fejdspar gneiss contaning up to 2 percent disseminated pyrite and maybe pythotite. \\
\hline $01 \overline{M B} \overline{3} \bar{B} \overline{8} \bar{A}$ & 483034 & $7 \overline{21100}$ & Eağle & $\bar{A} \overline{1}$ & Black quartzite containing trace to 2 percent disseminated pyrite and pyrite along foliation. \\
\hline $01 \mathrm{MB}=\overline{4} \overline{2} \overline{\mathrm{A}}$ & $49 \overline{440 \overline{3}}$ & $\overline{7} \overline{1} \overline{\bar{\gamma}} \overline{4} \overline{2} \overline{9}$ & Ėagle & $\vec{A} \dot{1}$ & Unknown original rock, now orange gossaneous material with no relict textures. \\
\hline $01 \mathrm{MBW} 427 \mathrm{~A}$ & 494502 & $7107 \overline{75}$ & Eagle & A1 & Select sample of tan to gray, aphanitic, chalcedonic silica veins cutting clay-iron-bearing carbonate. \\
\hline 01MBW429A & 493985 & 7107188 & Eagle & A1 & Fault breccia composed of gray colored aphanitic silica and $\uparrow$ inch diameter angular clasıs of unknown rock type. \\
\hline 01MBW430A & 493127 & $\grave{7105} 7 \mathbf{3 5}$ & Eagle & A1 & Gossaneous rock, possibly altered gneiss, with iron carbonate replacing rock and cut by vuggy quartz veins. \\
\hline$\overline{01} \overline{M B} \bar{B} \overline{3} \bar{B} \bar{A}$ & $49 \overline{9} \overline{0} \overline{4}$ & $710 \overline{7} 3 \overline{9}$ & Eagle & A1 & Aphanitic to banded gray silica breccia matrix with "clasts" of white crystalline calcite cut by silica-tron sulfide veins. \\
\hline $01 \mathrm{R} N 38 \mathrm{~A}$ & 488994 & 7103743 & Eagle & A1 & (Vein?) quartz boulders with approximately 1 percent disseminated sulfide (pyrite?). No other rock nearby. \\
\hline 01RN237B & 497451 & 7096886 & Eagle & A1 & Grab sample of grassy green, coarse-grained clinopyroxenite boulders up to $0.2 \mathrm{~m}$ wide. \\
\hline $01 Z 20 ̈ \mathrm{~A}$ & 485113 & 7102458 & Ëagle & $\mathrm{Ai}$ & Very fine-grained to sucratic, olive-brown uitramafic rock, looks microgabbroic with weathered olivine. \\
\hline $01 \overline{2} 20 B$ & 485113 & $7 \overline{10} 245 \overline{8}$ & Eagle & A1 & Dark green to black Littramafic rock with olivine and pyroxene, porphyritic phlogopite, and sprays of ?actinolite? \\
\hline $01233 \mathrm{C}$ & $48 \overline{8977}$ & 7107537 & Eagle & A1 & Select sample of pegmaite cut by vugs with drusy quartz and brown iron oxides occasional quariz vein. \\
\hline $01 \bar{Z} 2 \mathrm{~A}$ & 490707 & 7116101 & Eagle & $\overline{\mathrm{A}} \dot{1}$ & Coarse-grained amphibolite with randomly oriented crystals and minor epidote alteration. \\
\hline $01 Z 104 \mathrm{C}$ & 491234 & 7107683 & Eagle & A1 & Highly limonite stained quartz veins with extremely vuggy areas and subhedral quartz shapes. \\
\hline $01 Z 118 \mathrm{~A}$ & 495710 & 7116102 & Eagle & A1 & Bluish-gray biotite quartite with distinct banding, weak limonite stain, and common orangish quartz microveinlets. \\
\hline $012143 \mathrm{~A}$ & 495208 & 7103809 & Eagle & A1 & Orange to black iron stained faull breccia with white quartz veins. Sucratic white silica matrix with paie green clasts. \\
\hline $01 Z 152 B$ & 495863 & 7113286 & Eagle & At & Breccia with sucratic quartz matrix and quartzite clasts, trace amount of disseminated pyrite and abundant limonite. \\
\hline 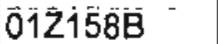 & $4 \overline{9} 50 \overline{9} 8$ & $\overline{7} 119 \overline{5} \overline{\overline{7}}$ & Eaglé & A1 & White quartz vein rubble and minor quartz vein breccia (clear angular quartz clasts in quartz matrix) with limonite. \\
\hline $01 Z 158 \mathrm{~F}$ & 495098 & 7119507 & Ëagle & Ā & Select sample of granite cut by vuggy, open-space quartz veins with iron-oxide stained vugs. \\
\hline
\end{tabular}




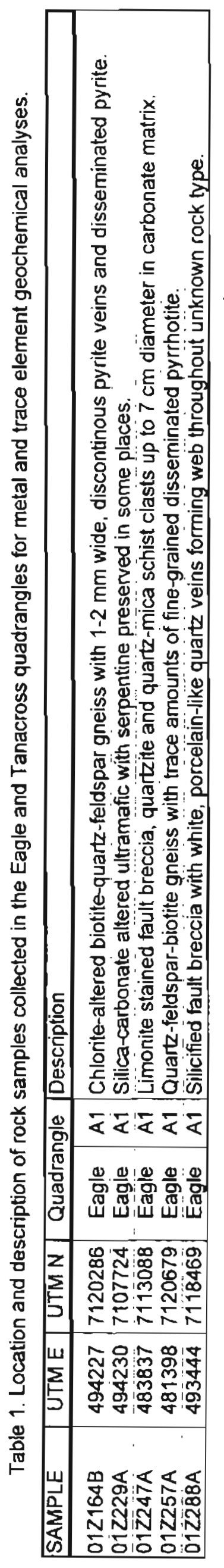


Table 2. Concentration of metals and trace elements in rock samples from the Eagle and Tanacross quadrangles, Alaska. Note: - = not analyzed.

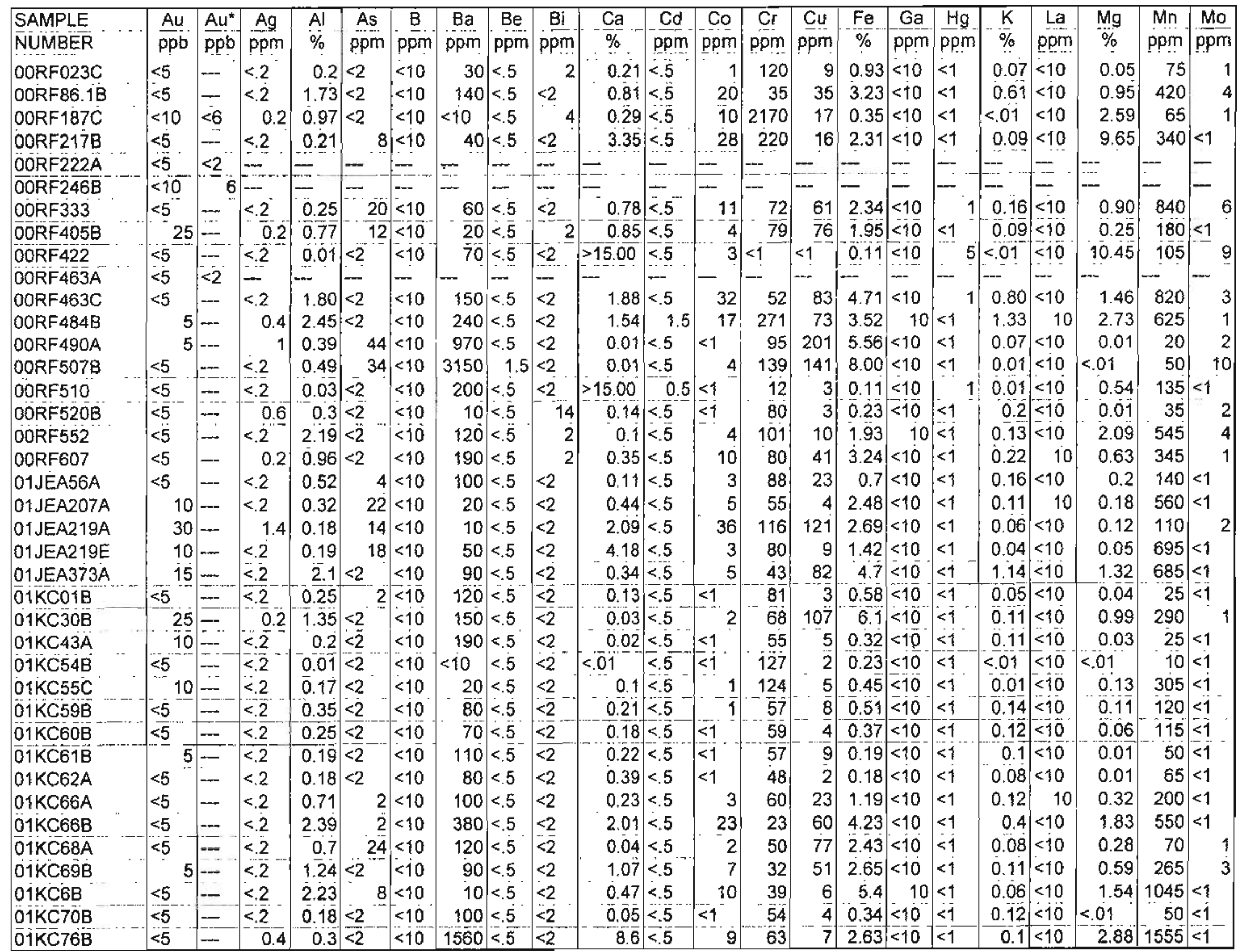


Table 2. Concentration of metals and trace elements in rock samples from the Eagle and Tanacross quadrangles, Alaska. Note; -- = not analyzed.

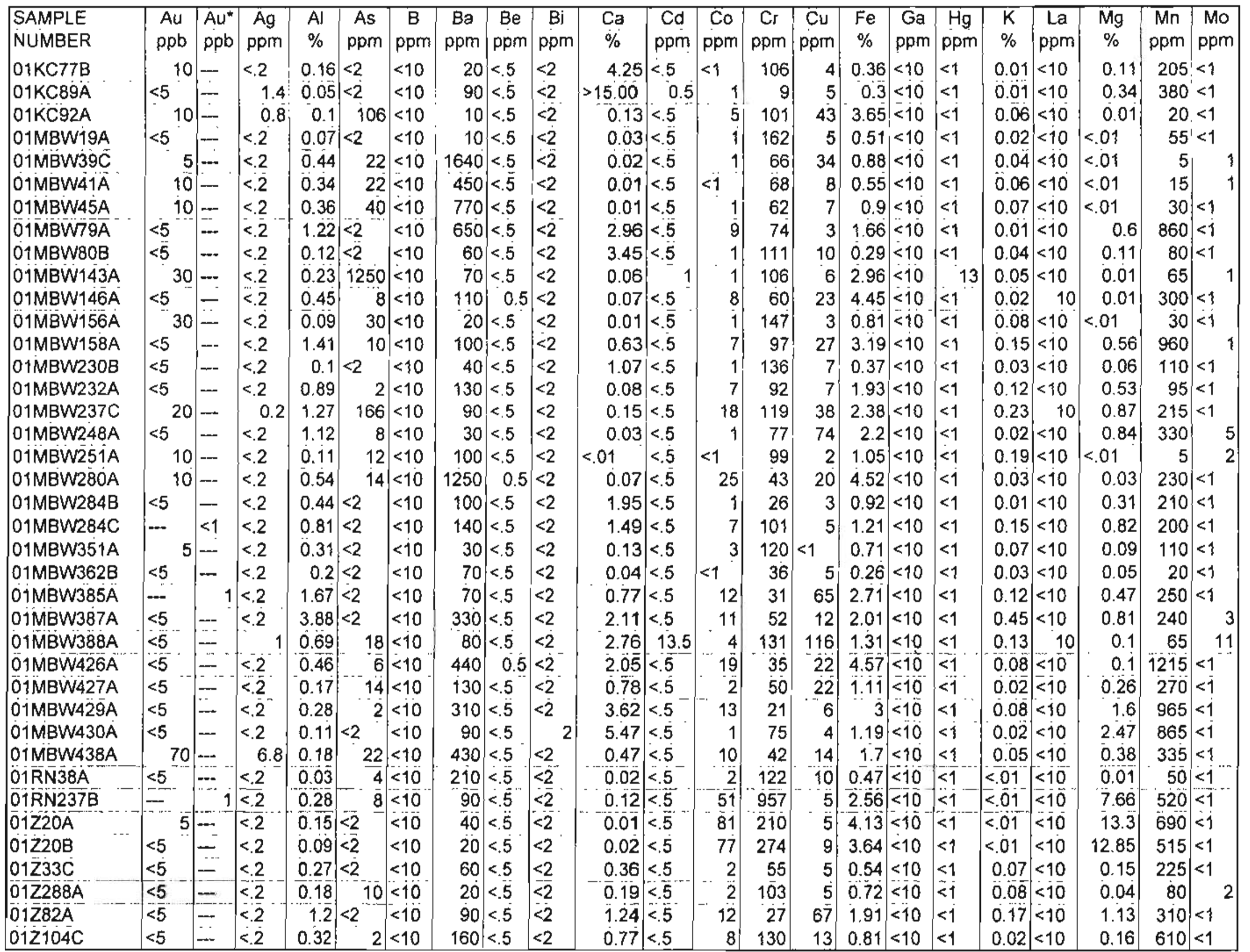




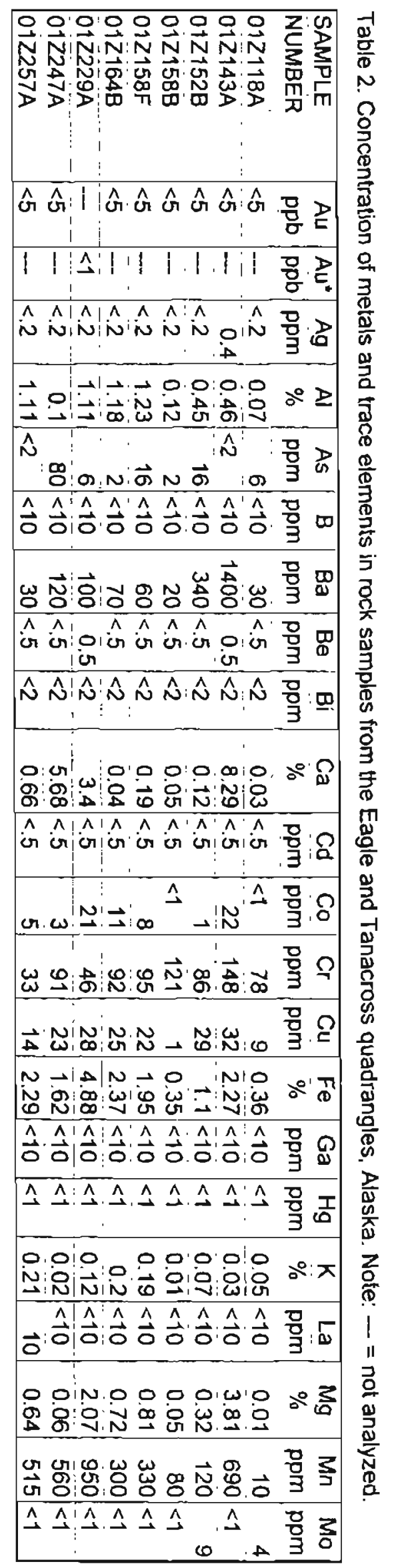


Table 2. Concentration of metals and trace elements in rock samples from the Eagle and Tanacross quadrangles, Alaska. Note: *- = not analyzed

\begin{tabular}{|c|c|c|c|c|c|c|c|c|c|c|c|c|c|c|c|c|c|}
\hline $\begin{array}{l}\text { SAMPLE } \\
\text { NUMBER }\end{array}$ & & $\begin{array}{l}\mathrm{Na} \\
\%\end{array}$ & $\begin{array}{c}\mathrm{Ni} \\
\mathrm{ppm}\end{array}$ & $\begin{array}{c}\mathrm{P} \\
\mathrm{ppm}\end{array}$ & $\begin{array}{l}\mathrm{Pb} \\
\mathrm{Ppm}\end{array}$ & $\begin{array}{l}\mathrm{S} \\
\%\end{array}$ & $\begin{array}{c}\mathrm{Sb} \\
\mathrm{ppm}\end{array}$ & $\begin{array}{c}\mathrm{Sc} \\
\mathrm{ppm}\end{array}$ & $\begin{array}{c}\mathrm{Sr} \\
\mathrm{ppm}\end{array}$ & $\begin{array}{l}\text { Ti } \\
\%\end{array}$ & $\begin{array}{c}\mathrm{TI} \\
\mathrm{ppm}\end{array}$ & $\begin{array}{c}\mathrm{U} \\
\mathrm{ppm}\end{array}$ & $\begin{array}{c}\mathrm{V} \\
\mathrm{ppm}\end{array}$ & $\begin{array}{c}\text { W } \\
\text { ppm }\end{array}$ & $\begin{array}{c}\mathrm{Zn} \\
\mathrm{ppm}\end{array}$ & $\begin{array}{c}\mathrm{Pt} \\
\mathrm{ppb}\end{array}$ & $\begin{array}{c}\mathrm{Pd} \\
\mathrm{ppb}\end{array}$ \\
\hline OORF023C & & $<.01$ & 6 & 260 & 6 & $<.01$ & $<2$ & 2 & 4 & $<.01$ & $<10$ & $<10$ & 5 & $<10$ & 40 & - & $\because$ \\
\hline 0ORFB $1 \mathrm{~B}$ & & 0.04 & 17 & 1500 & 8 & 0.37 & $<2$ & 5 & 39 & 0.11 & 10 & $<10$ & 56 & $<10$ & 60 & - & $\cdots$ \\
\hline $00 R F 187 \mathrm{C}$ & & 0.01 & 578 & $<10$ & $<2$ & $<.01$ & 8 & $<1$ & 16 & $<.01$ & $<10^{-}$ & $<10$ & 11 & $<10$ & 6 & $<6$ & $<6$ \\
\hline OORF217B & & $<.01$ & 379 & 20 & $<2$ & $<.01$ & $<2$ & 4 & 100 & $<.0 \hat{1}$ & $<10$ & $<10$ & 8 & $<10$ & 16 & - & -- \\
\hline DORF222A & & |-- & - & - & -- & - & -- & --- & - & -- & - & - & - & --- & - & $<5$ & $<2$ \\
\hline OORF246B & & $\cdots$ & $\ldots$ & $\cdots$ & - & -- & - & $\cdots$ & --- & -- & $-\cdots$ & - & - & - & -- & $<6$ & $<6$ \\
\hline OORF333 & & 0.01 & 64 & 200 & 6 & 1.04 & $<2$ & 2 & 17 & $<01$ & 20 & $\mid<10$ & 10 & $<10$ & 40 & $\ldots$ & $\cdots$ \\
\hline OORF405B & & 0.07 & 5 & 120 & $<2$ & 0.35 & $<2$ & 2 & 15 & $<.01$ & $<10$ & $<10$ & 12 & $<10$ & 18 & -- & - \\
\hline OORF 422 & & 0.01 & & 50 & 4 & $<.01$ & $<2$ & $<1$ & 117 & $<01$ & $<10$ & $<10$ & 4] & $<10$ & 12 & - & $=$ \\
\hline OORF 463A & & - & & - & - & - & - & - & -- & $-\infty$ & --- & $\because$ & -1 & $-\infty$ & - & $<5$ & $<2$ \\
\hline OORF463C & & 0.13 & 30 & 4470 & 2 & 1.23 & $<2$ & 6 & 48 & 0.18 & 10 & $<10$ & 92 & $<10$ & 30 & - & - \\
\hline OORF484B & & 0.06 & 42 & 560 & 8 & 0.93 & $<2$ & 15 & 33 & 0.16 & $<10$ & $<10$ & 104 & $<10$ & 76 & $-\infty$ & - \\
\hline OORF $490 \mathrm{~A}$ & & 0.01 & 5 & 160 & $1 \ddot{4}$ & 0.04 & 38 & $<1$ & 11 & $<.01$ & $<10$ & $<10$ & 29 & $<10$ & 12 & - & - \\
\hline OORF507B & & 0.01 & 19 & 3490 & $<2$ & 0.06 & 6 & 1 & 42 & $<.01$ & $<10^{\circ}$ & $<10$ & 145 & $<10$ & 172 & - & - \\
\hline OORF510 & & 0.01 & 1 & 110 & $<2$ & 0.04 & $<2$ & $<1$ & 374 & $<.01$ & $<10$ & $<10$ & 2. & $<10$ & 8 & - & - \\
\hline OORF $520 \mathrm{~B}$ & & 0.05 & 2 & 570 & 16 & $<01$ & $<2$ & $<1$ & 1 & $<.01$ & $<10$ & $<10$ & $<1$ & $<10$ & 6 & -- & - \\
\hline $00 R F 552$ & & 0.02 & 22 & 550 & $3 \overline{2}$ & 0.02 & $<2$ & 1 & 8 & $<.01$ & $<10$ & $<10$ & 17 & $\mid<10$ & 96 & - & -- \\
\hline OORF607 & & 0.01 & 55 & 710 & 2 & 0.01 & 2 & 2 & 11 & $<.01$ & $<10^{\circ}$ & $<10$ & 20 & $<10$ & 98 & $-\ldots$ & - \\
\hline 01 JEA56A & & 0.08 & 5 & 70 & 10 & $<.01$ & $<2$ & 1 & 19 & 0.01 & $<10$ & $<10$ & 7 & $\mid<10$ & 12 & - & - \\
\hline 01JEA207A & & 0.05 & 9 & 180 & $<2$ & $<.01$ & 2 & 7 & 10 & 0.02 & $<10$ & $<10$ & 18 & $<10$ & 42 & - & - \\
\hline $01 \mathrm{JEA} 219 \mathrm{~A}$ & & $<01$ & 14 & 100 & $\overline{<2}$ & 2.92 & 18 & $<1$ & 26 & 0.01 & $<j 0$ & $<10$ & 6 & $<10$ & 6 & - & - \\
\hline $01 \mathrm{JEA} 219 \mathrm{E}$ & & $<01$ & 6 & 480 & $<2$ & 1.43 & 10 & 1 & 70 & $<.01$ & $<10^{\circ}$ & $<10$ & 4 & $<10$ & 14 . & -. & $\ldots$ \\
\hline 01JEA373A & & 0.04 & 1 & 1090 & 2 & 0.96 & 4 & 10 & 8 & 0.16 & $<10$ & $<10$ & 10 & $<10$ & 386 & -- & $\ldots$ \\
\hline $01 \mathrm{KCO} 1 \mathrm{~B}$ & & 0.13 & 4 & 60 & $\overline{6}$ & 0.11 & $<2$ & $<1$ & $2 \overline{1}$ & $<.01^{\circ}$ & $<10$ & $<10$ & $<1$ & $<10$ & $\{0$ & $=$ & $m$ \\
\hline $01 \mathrm{KC} 30 \mathrm{~B}$ & & 0.07 & 9 & 380 & 2 & 0.3 & 6 & 8 & 24 & 0.03 & $<10$ & $<10$ & 78 & $<10$ & 60 & -- & - \\
\hline $01 \mathrm{KC} 43 \mathrm{~A}$ & & 0.06 & $\dagger$ & 30 & 2 & $<.01$ & $<2$ & $<1$ & 18 & $<.01$ & $<10$ & $<10$ & 4 & $<10$ & $<2$ & - & - \\
\hline $01 \mathrm{KC} 54 \mathrm{~B}$ & & $<.01$ & 3 & $<10$ & $<2$ & $<.01$ & $<2$ & $<1$ & $<1$ & $<.01$ & $<10$ & $<10$ & $<1$ & $<10$ & $<2$ & - & - \\
\hline $01 \mathrm{KC5} 5 \mathrm{C}$ & & $<.01$ & 4 & 30 & $<2$ & $<.01$ & $<2$ & $<1$ & 5 & $<.01$ & $<10$ & $1<10$ & 4 & $<10$ & $<2$ & - & - \\
\hline 01kC59B & & 0.09 & 1 & 70 & 2 & $<.01$ & $<2$ & $<1$ & 26 & 0.01 & $<10$ & $<10$ & 8 & $<10$ & 16 & - & - \\
\hline$\overline{0} \overline{\mathrm{K}} \overline{\mathrm{C}} \overline{\mathrm{6}} \overline{\mathrm{O}} \overline{\mathrm{B}}$ & & 0.07 & 1 & 30 & $<2$ & $<.01$ & $<2$ & $<1$ & 23 & $<.01$ & $<10^{\circ}$ & $<10$ & 5 & $<10$ & 6 & - & - \\
\hline 01KC61B & & 0.08 & 1 & 10 & $<\overline{2}$ & 0.01 & $<2$ & $<1$ & 28 & $<.01$ & $<10$ & $<10$ & 1 & $<10$ & $<2$ & $\ldots$ & $m$ \\
\hline $01 \mathrm{KC} 62 \mathrm{~A}$ & & 0.08 & 1 & $<10$ & 2 & 0.02 & $1<2$ & $<1$ & 38 & $<.01$ & $<10$ & $<10$ & $<1$ & $i<10$ & $<2$ & - & $\ldots$ \\
\hline $01 \mathrm{KC} 66 \mathrm{~A}$ & & 0.06 & 5 & 360 & 6 & 0.08 & 2 & 2 & 20 & 0.04 & $<10$ & $<10$ & 15 & $<10$ & 28 & - & - \\
\hline $01 \mathrm{KC} 66 \mathrm{~B}$ & & 0.17 & 8 & $3 \overline{120}$ & $<2$ & 0.14 & 4 & $1 \overline{1}$ & 71 & 0.21 & $<10$ & $<10$ & $13 \overline{2}$ & $<10$ & $6 \overline{6}$ & - & - \\
\hline C68A & & 0.07 & 8 & 120 & 28 & 0.06 & 2 & 3 & 7 & $<.01$ & $<i 0$ & $<10$ & 18 & $<10$ & 58 & - & - \\
\hline $6 \mathrm{C} 69 \mathrm{~B}$ & & 0.08 & 4 & 1190 & $<2$ & 0.14 & 2 & 4 & 61 & 0.1 & $<10$ & $<10$ & 47 & $<10$ & 46 & - & -- \\
\hline C6日 & & 0.07 & 1 & 2150 & $<2$ & 0.56 & 6 & 13 & 7 & 0.04 & $<10$ & $<10$ & 72 & $<10$ & 106 & -- & $m$ \\
\hline $01 \mathrm{KC} 70 \mathrm{~B}$ & & 0.07 & 1 & 30 & 2 & 0.04 & $<2$ & $<1$ & 20 & $|<.04|$ & $<10$ & $<10$ & 1 & $<10$ & 2 & - & -_- \\
\hline $01 \mathrm{KC} 76 \mathrm{~B}$ & & 0.01 & 3 & 10 & $<2$ & 0.04 & 2 & -6 & $25 \overline{6}$ & $<.01$ & $<10$ & $<1 \overline{0}$ & 18 & $<10$ & $3 \overline{0}$ & E- & 二- \\
\hline
\end{tabular}




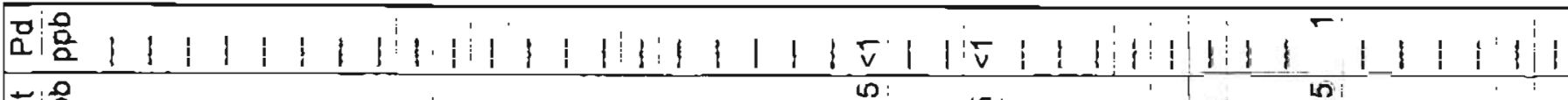

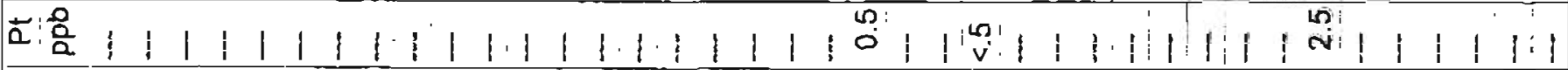

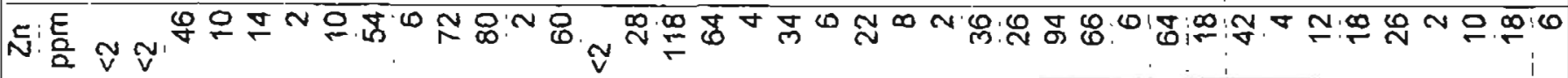

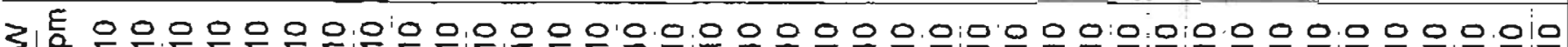

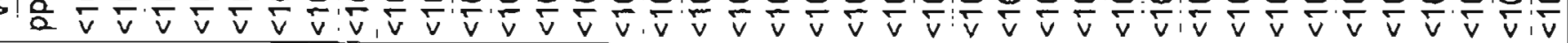

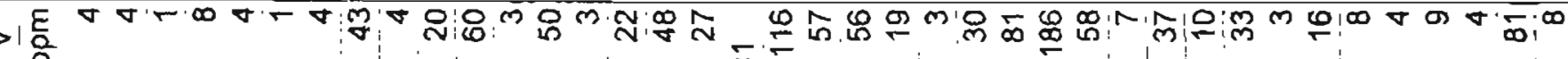
$\bar{v}-$

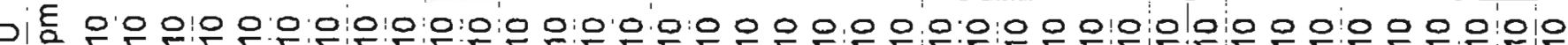

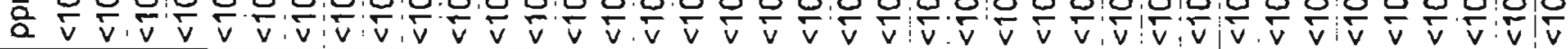

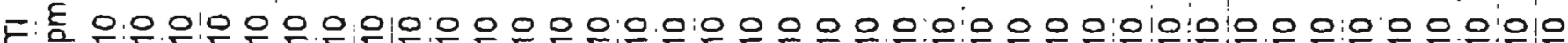

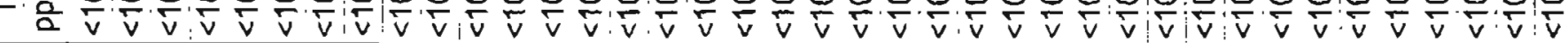

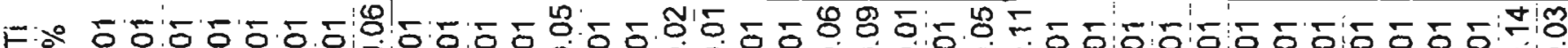

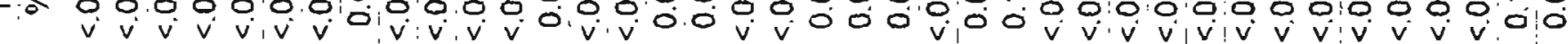
দ

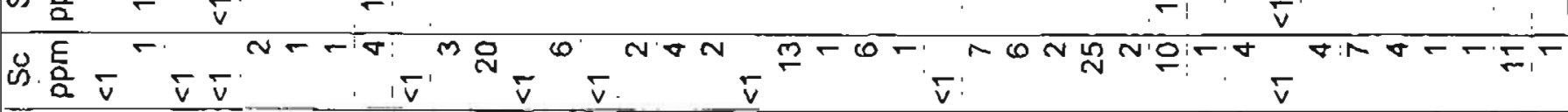

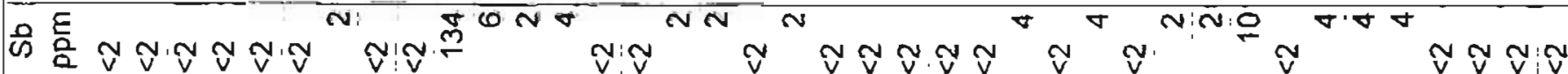

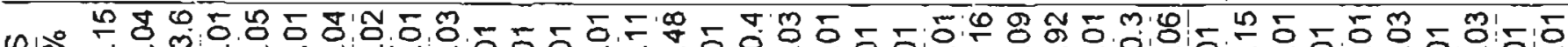

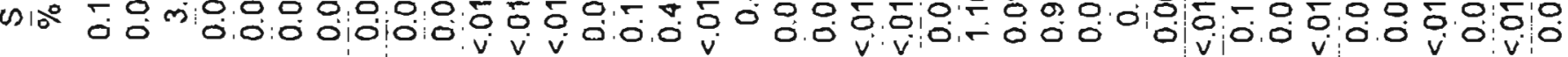

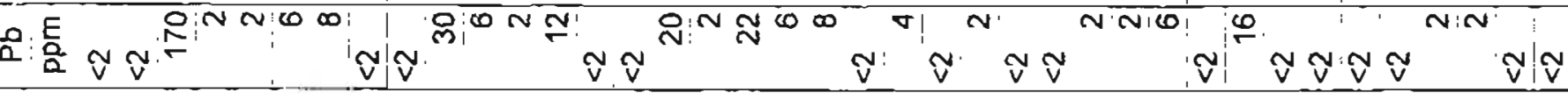
ㄷ. 틍

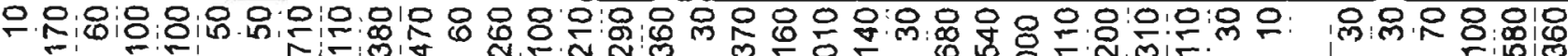

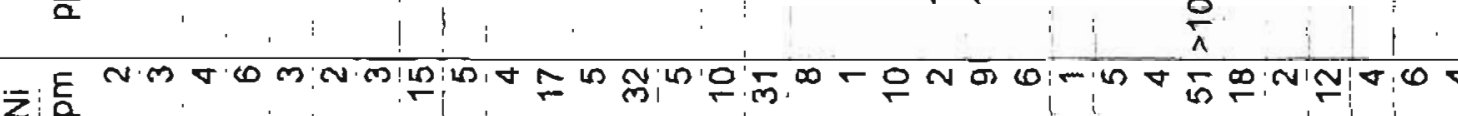
Zi $\frac{a}{2}$

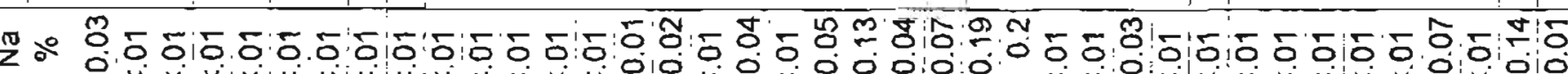

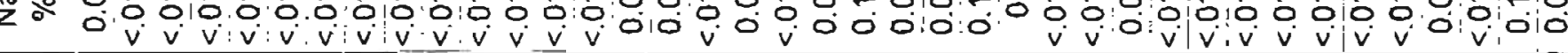

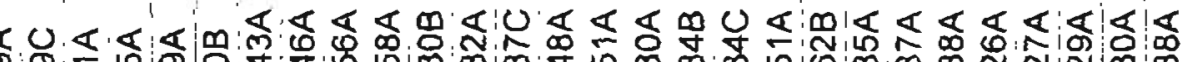

w年

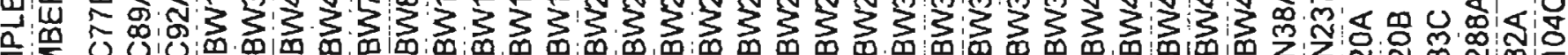

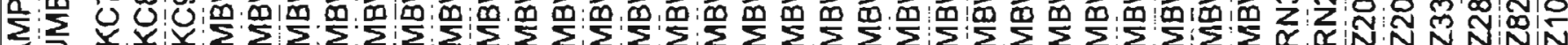
造 


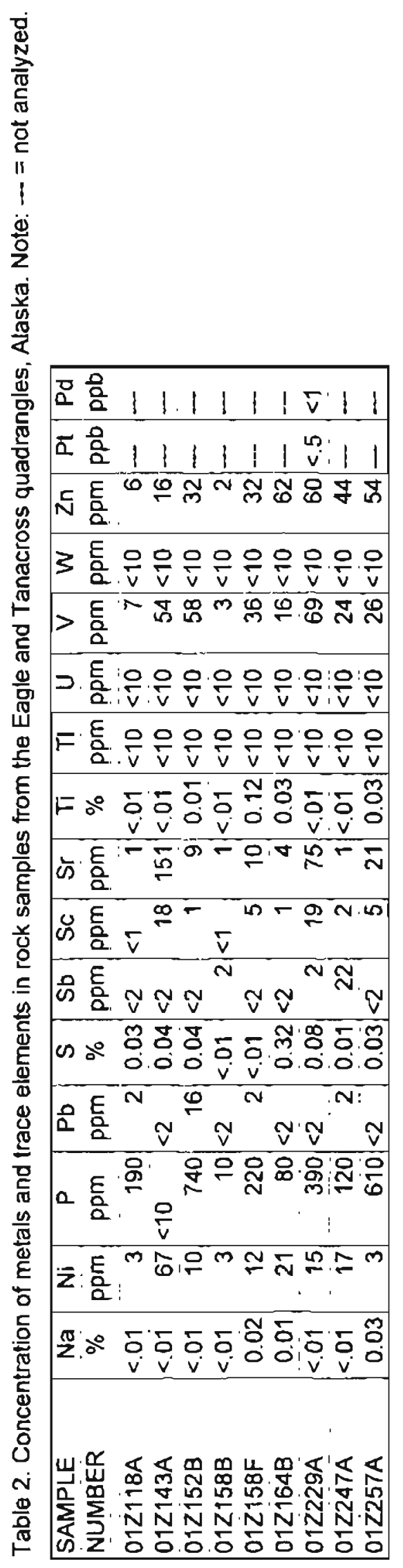


Table 3. Location and description of rock samples collected in the Eagle and Tanacross quadrangles for major-oxide, minor oxide, and trace eiement analyses.

\begin{tabular}{|c|c|c|c|c|c|c|}
\hline SAMPLE & UTME & UTM N & \multicolumn{2}{|c|}{ Quadrangle } & \multicolumn{2}{|l|}{ Rock Type } \\
\hline$\overline{99 M B W} \overline{7} 9$ & 451832 & 7105237 & Eagle & $\mathrm{A}-2$ & Finely crystalline, siliceous tuff & \\
\hline 99MBW095̄B & $4 \overline{49900}$ & 7106867 & Eagle & A-3 & Tuff, possibly airfall or waterlain & \\
\hline OODS121 & 471660 & 7111369 & Eagle & $\mathrm{A}-2$ & Amphibolite & \\
\hline 00JG014-6/28 & 482139 & 7107044 & Eagle & A-1 & Amphibolite & \\
\hline O0JG032-6/29 & 479359 & 7107661 & Eagle & A-1 & Biotite amphibolite & \\
\hline 00JG092-6/20 & 479862 & 7108898 & Eagle & A-1 & Fine-grained garnet amphibolite & \\
\hline $00 . j G 095-6 / 20$ & 479177 & 7108615 & Eagle & $A-1$ & Amphibolite & \\
\hline OOMBN194 & 464886 & 7112185 & Eagle & $\mathrm{A}-2$ & White, fine-grained felsic plutonic rock & \\
\hline OOMBW213C & 455857 & 7116727 & Eagle & A-2 & Granitic dike & \\
\hline OOMBW535 & 463440 & $712007 \overline{7}$ & Eagle & $\mathrm{A}-2$ & Granite or granodiorite & \\
\hline OOMBW549 & 462333 & $712025 \overline{7}$ & Ëaglè & $\mathrm{A}-2$ & Aplite dike & \\
\hline OORFOOIA & 498192 & 7105642 & Eagle & $\mathrm{A}-1$ & Biotite-chlorite-quartz-white mica schist & \\
\hline OORFO54 & 497233 & 7101488 & Eagle & A-1 & Biotite-quartz-feldspar schist & \\
\hline 00RF061 & 495973 & 7102236 & Eağle & $\mathrm{A}-1$ & Älbite-chlorite-actinolite-clinozoisite metagabbro & \\
\hline DORF061 & 495973 & 7102236 & Eagle & $\mathrm{A}-1$ & Albite-chlorite-actinolite-clinozoisite metagabbro & \\
\hline ÖORF092A & 497122 & 7096261 & Tanacross & D-1 & White mica-albite-actinolite-chlorite-clinozoisite metagabbro & \\
\hline $00 R F 092 \bar{A}$ & 497122 & $7 \overline{0} 96261$ & Tanacross & $\mathrm{D}-1$ & White mica-albite-actinolite-chlorite-clinozoisite metagabbro & $\ldots$ \\
\hline OORF112A & $49 \overline{7} \overline{3} \overline{2}$ & 7094053 & Tanacross & D-1 & Amphibolite & 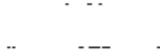 \\
\hline OORF $1 \uparrow 3 \mathrm{~B}$ & $\overline{4} 9 \overline{7} \overline{427}$ & 7093765 & Tanacross & D-1 & Lithic rhyolite & \\
\hline OORF 118 & $49737 \dot{8}$ & 7093262 & Ṫanacross & $\mathrm{D}-1$ & Muscovite-K feldspar-biotite-plagioclase-quart \pm garnet schist & \\
\hline $\bar{O} \bar{R} \bar{F}+2 \overline{6} \mathrm{~A}$ & 496475 & 7092511 & Tanacross & D-1 & Garnet-biotite-clinozoisite-plagioclase-muscovite-quartz schist & \\
\hline $0 O \overline{R F}\{\overline{2} 6 \bar{A}$ & 496475 & 7092511 & Tanacross & D-1 & Gamet-biotite-clinozoisite-plagioclase-muscovite-quartz schist & \\
\hline OORF126B & 496435 & 7092511 & Tanacross & D-1 & Garnel-bearing amphibolite & \\
\hline OORF134 & 496337 & 7102823 & Eagle & $A-1$ & Albite-chlorite-actinolite-clinozoisite metagabbro & \\
\hline oORF134 & 496337 & 7102823 & Eagle & A-1 & Albite-chlorite-actinolite-clinozoisite metagabbro & \\
\hline $00 R F 160$ & 491220 & $7097 \overline{7} 25$ & Eagle & A-1 & Biotite-K feldspar-muscovite-quartz schist & \\
\hline OORF 174A & 497282 & 7099914 & Eagle & A-1 & Clinozoisite-biotite-homblende-plagioclase-quartz gneiss & \\
\hline 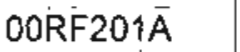 & 496792 & 7101221 & Eagle & A-1 & Clinozolsite-chlorite-K feldspar-white mica-albite-quartz metafelsite & \\
\hline OORF236A & 497486 & 7098634 & Eagle & A-1 & Biotite- epidote amphibolite & \\
\hline$\overline{0 O R F} \overline{3} 2 \overline{2 A}$ & 496271 & 7102840 & Eagle & $\mathrm{A}-1$ & Albite-actinolite-clinozoisite metagabbro & $\ldots$. \\
\hline OORF340B & 496947 & 7103915 & Eagle & A-1 & Kyanite quartzite & \\
\hline OORF345A & 497584 & 7103551 & Eagle & $A-1$ & Biotite-chlorite-albite-clinozoisite-actinolite metagabbro & \\
\hline OORF349A & 498795 & 7104505 & Eagle & A-1 & Biotite-chlorite-actinolite schist & \\
\hline OORF349B & 498795 & 7104505 & Eagle & A-1 & Fuchsite-quartz-actinolite schist & \\
\hline 00RF357C & 498905 & 7103822 & Eagle & A-1 & Biotite-chlorite-actinolite schist & \\
\hline OORF376 & 493871 & 7104480 & Eagle & A-1 & Gatnet-bearing amphibolite & \\
\hline $0 O \mathrm{RF} 454$ & 498817 & 7092589 & Tanacross & $\mathrm{D}-1$ & Biotite-muscovite-feldspar-quartz schist and quartzite & \\
\hline $0 \overline{O O R} \overline{4} \overline{6} \overline{7}$ & 495900 & $70946 \overline{6} \overline{0}$ & Tanacioss & $\mathrm{D}-1$ & Amphibolite & \\
\hline OORF $487 \mathrm{~B}$ & 497157 & 7097544 & Eagle & $A=1$ & Rhyolite dike or sill & \\
\hline
\end{tabular}


Tabie 3. Location and description of rock samples collected in the Eagle and Tanacross quadrangles for major-oxide, minor oxide, and trace element analyses.

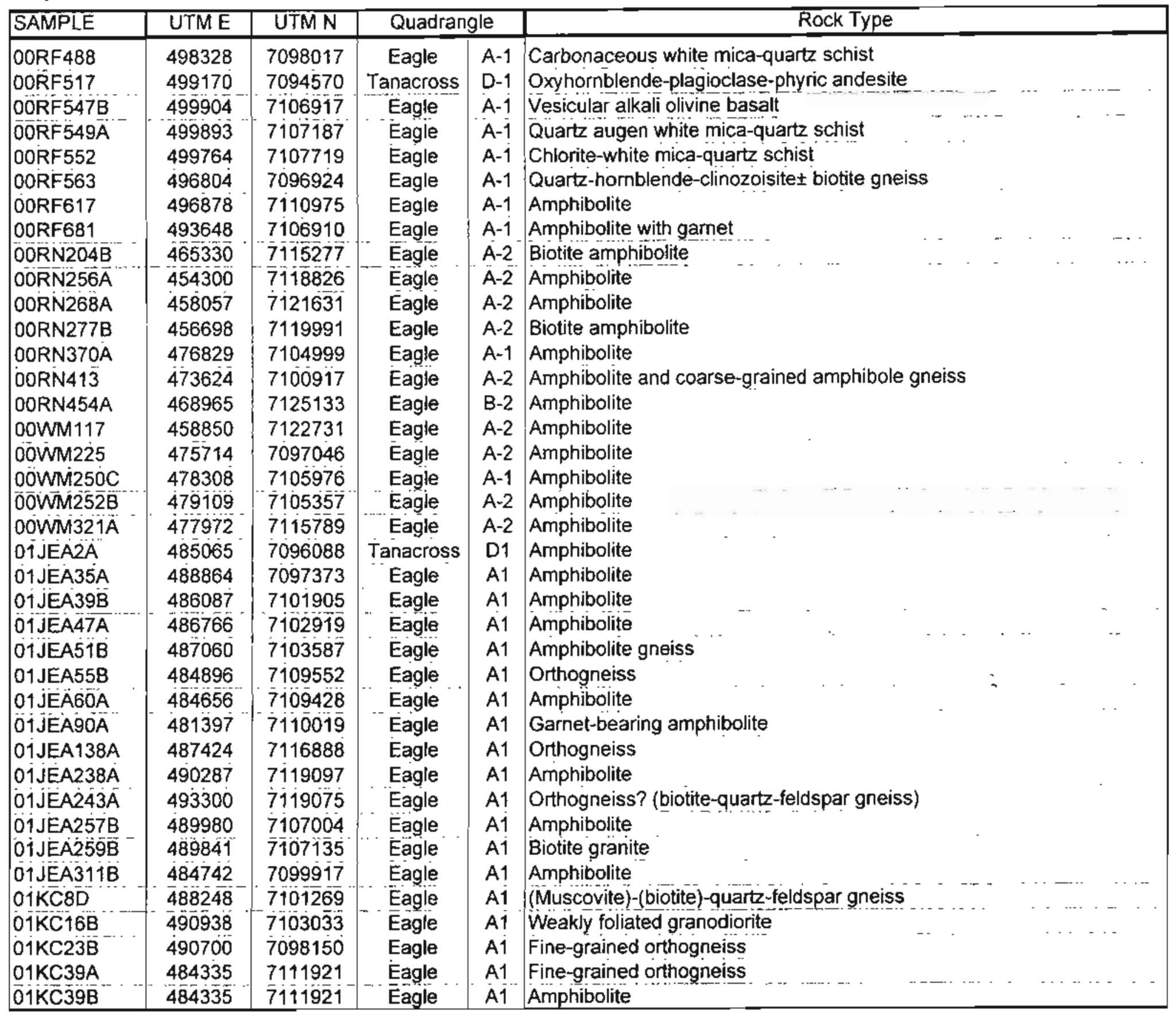


Table 3. Location and description of rock samples coltected in the Eagle and Tanacross quadrangles for major-oxide, minor oxide, and irace element analyses.

\begin{tabular}{|c|c|c|c|c|c|}
\hline SAMPLE & UTME & UTMN & Quadra & & Rock Type \\
\hline $01 \mathrm{KC} 41 \mathrm{~A}$ & 485485 & 7109782 & Eagle & A1 & Slightly foliated granodiorite dike \\
\hline $01 \mathrm{KC} 42 \mathrm{~A}$ & 485715 & 7109963 & Eagle & A1 & Biotite granodionite \\
\hline $\mathrm{C} 47 \mathrm{~B}$ & 485451 & 7115741 & Eagle & A1 & Biotite-quartz-feldspar gneiss \\
\hline $51 \mathrm{~B}$ & 488029 & 7119717 & Eagle & A1 & Biotite orthogneiss \\
\hline $5 \bar{A}$ & 494579 & 7109437 & Eagle & $\mathrm{A} 1$ & Amphibolite \\
\hline $58 \mathrm{~A}$ & 494826 & 7110870 & Eagle & A1 & Biotite-hornblende gabbro \\
\hline $69 A$ & 494088 & $7113 \overline{359}$ & Éagle & $\mathrm{A} 1$ & Slighty foliated biotite granodiorite \\
\hline $1 \mathrm{~A}$ & 494085 & 7113993 & Eagle & A1 & Slightly foliated biotite granodiorite \\
\hline $01 \mathrm{KC} 66 \mathrm{~B}$ & $494 \overline{8} 4 \overline{2}$ & $\overline{7110688}$ & Eagle & A1 & Amphibolite \\
\hline $59 B$ & 494095 & 7112905 & Eagle & A1 & Highty foliated homblende-biotite granodiorite \\
\hline $01 \mathrm{KC95C}$ & 493248 & 7125954 & Eagle & B1 & Fine-grained felsic dikes \\
\hline $01 \mathrm{KC95D}$ & 493248 & 7125954 & Eagle & B1 & Fine-grained ultramafic (biotite clinopyroxenite?) \\
\hline W10A & 484758 & 7097374 & Eagle & A1 & White mica-bearing orthogneiss \\
\hline Wi4A & 484559 & 7098202 & Eagle & A1 & Ortho \\
\hline N50A & 492040 & 7102 & Eagle & A1 & Thinly foliated, biotite orthogneiss \\
\hline N52A & 492415 & 7102 & Eagle & A1 & amphibolite \\
\hline W57B & 485417 & 7102598 & Eagle & A1 & Amphibolite \\
\hline WGOA & 485380 & 7103 & Eagle & A1 & White mica-bearing ofthogneiss \\
\hline 01MBW63А & 485264 & 7103538 & Eagle & A1 & phibolite \\
\hline W73A & 486159 & 7104629 & Eagle & A1 & Porphyritic basalt \\
\hline W77B & 484886 & 7103 & Eagle & A1 & White mica-bearing orthogneiss \\
\hline W95B & 487383 & 710 & Eagle & A1 & Chiorite orthogneiss \\
\hline W105B & 488 & 710 & Eagle & A1 & Weakly foliated, biotite tonalite $\ldots .$. \\
\hline $01 \mathrm{MBW} 123 \mathrm{~A}$ & 485561 & 7116 & Eagle & A1. & Tonalitic to granodioritic orthogneiss. \\
\hline $01 \mathrm{MBW} 125 \mathrm{~A}$ & 485657 & 711 & Eagle & A1 & Granitic, biotite orthognelss \\
\hline $\mathrm{V} 126 \mathrm{~A}$ & & & Eagle & A1 & Fine-grained, while mica-bearing gneiss \\
\hline W130A & 097 & & Eagle & A1. & Foliated, granitic, homblende-bearing plutonic rock \\
\hline $01 \mathrm{MBW} 134 \mathrm{~A}$ & & 711 & Eagle & A1 & Coarse-grained pegmakite \\
\hline D1MBW144A & & & Eagle & A? & Weakly foliated, fine-grained, biotite granodiorite \\
\hline $01 M B W 165 A$ & & & Eagle & A1 & Slightly foliated, granitic biotite orthogneiss \\
\hline $01 \mathrm{MBW} 171 \mathrm{~A}$ & & & Eagle & At & Amphibolite \\
\hline $01 \mathrm{MBW} 180 \mathrm{~A}$ & & & Eagle & A1 & Granite \\
\hline 01MBW183A & & & Eagle & A1 & White mica-biotite-quartz-feldspar orthogneiss \\
\hline 01MBW187A & & & Eagle & A1 & Gamet-bearing amphibolite \\
\hline $01 \mathrm{MBW} 225 \mathrm{~A}$ & & 7119820 & Eagle & A1 & Slightly foliated, tonalitic, biotite orthogneiss \\
\hline $01 \mathrm{MBW} 226 \mathrm{~B}$ & 493250 & & Eagle & A1 & Amphibolite \\
\hline 01MBW244A & & 7113298 & Eagle & A1 & Orthogneiss or metaconglomerate \\
\hline $01 \mathrm{MBW} 245 \mathrm{~A}$ & 496062 & 7113303 & Eagle & A1. & Meta-pebble sandstone or metavolcanic rock. \\
\hline $01 \mathrm{MBW} 246 \mathrm{~B}$ & 496020 & 7113310 & Eagle & A1 & Amphibolite \\
\hline
\end{tabular}


Tabie 3. Location and description of rock samples collected in the Eagle and Tanacross quadrangles for major-oxide, minor oxide, and trace element analyses.

\begin{tabular}{|c|c|c|c|c|c|c|}
\hline SAMPLE & UTME & UTMN & \multicolumn{2}{|c|}{ Quadrangle } & \multicolumn{2}{|l|}{ Rock Type } \\
\hline $01 \mathrm{MBW} 252 \mathrm{~A}$ & 495356 & 7113359 & Eagle & A1 & Metagabbro & \\
\hline $01 \mathrm{MBW} 255 \mathrm{~A}$ & 485537 & 7122352 & Eagle & A1 & Biotite granodiorike dike & \\
\hline $01 \mathrm{MBW} 287 \mathrm{~B}$ & 491702 & 7106318 & Eagle & A1 & Biotite amphibolite. & \\
\hline $01 M B W 288 \mathrm{~A}$ & 491500 & 7106400 & Eagle & A1 & Biotite tonalite or orthogneiss & \\
\hline $01 M B W 289 \mathrm{~A}$ & 491328 & 7106576 & Eagle & A1 & Amphibolite & \\
\hline $01 \overline{\mathrm{B}} \mathrm{W} 2 \overline{\mathrm{A}} \mathrm{A}$ & $49027 \overline{3}$ & 7106862 & Eagle & A1 & White mica-biotite-quartz-feldspar orthogneiss & \\
\hline $01 \mathrm{MBW} 304 \mathrm{~A}$ & 499603 & 7122956 & Eagle & A1 & Amphibolite & \\
\hline 01MBW313A & 499530 & 7122081 & Eagle & A1 & Biotite-quartz-feldspar gneiss & \\
\hline $01 \mathrm{MBW} 314 \mathrm{~A}$ & 499282 & 7122042 & Eagle & A1 & Amphibolite & \\
\hline 01MBW319A & 498555 & 7121989 & Eagle & A1 & Amphibolite & \\
\hline $01 M B W 321 A$ & 497159 & 7121787 & Eagle & A1 & White mica-bearing orthogneiss & \\
\hline $01 M B W 327 \mathrm{~A}$ & 497934 & 7123708 & Eagle & A1 & White mica-bearing orthogneiss & \\
\hline $01 \mathrm{MBW} 330 \mathrm{~A}$ & 496727 & 7124032 & Eagle & A1 & Amphibole-feldspar gneiss & \\
\hline 01MBW335A & 495017 & 7119640 & Eagle & At & Slightly foliated gabbro & \\
\hline 01MBW336A & 494844 & 7119734 & Eagle & A1 & Biolite-hornblende-quartz-feldspar orthogneiss & \\
\hline 01MBW344A & 494346 & 7121018 & Eagle & A1 & Amphibolite & \\
\hline $01 \mathrm{MBW} 367 \mathrm{~A}$ & 480627 & 7098608 & Eagle & A1 & Tonalitic or trondhjemitic, white mica-bearing orth & ogneiss dike or sill \\
\hline 01MBW371A & 481412 & 7098989 & Eagle & A1 & Pegmatitic, biotite-bearing orthogneiss & \\
\hline 01MBW376A & 483234 & 7119461 & Eagle & A1 & Biotite granodioritic (?) orthogneiss & \\
\hline 01MBW377B & 483058 & 7119640 & Eagle & A1 & Porphyritic biotite-homblende monzonite (dike?) & \\
\hline 01MBW381B & 482972 & 7120238 & Eagle & A1 & Slightly foliated, biotite monzonite & \\
\hline 01MBW383A & 482964 & 7120551 & Eagle & A1 & White mica-bearing orthogneiss & \\
\hline 01MBW402A & 482004 & 7120580 & Eagle & A1 & Fine-grained, biotite-bearing? Amphibolite & \\
\hline 01MBW403A & 482081 & 7120862 & Eagle & A1 & Granodioritic, biotite orthogneiss & \\
\hline 01MBW418A & 487391 & 7121431 & Eagle & A 1 & Granodioritic, biotite orthogneiss & \\
\hline 01MBW432A & 493542 & 7106770 & Eagle & A1 & Amphibolite & \\
\hline 01MBW433A & 493682 & 7106916 & Eagle & A1 & Biotite amphibolite & \\
\hline $01 \mathrm{MBW} 436 \mathrm{~A}$ & 493918 & 7107213 & Eagle & A1 & White mica-bearing granite sill & \\
\hline O1RN13A & 480015 & 7099751 & Eagle & A1 & Biotite amphibolite & \\
\hline 01RN22A & 486905 & 7101189 & Eagle & A1 & Biotite-bearing amphibolite & \\
\hline 01RN46A & 482430 & 7100811 & Eagle & A1 & Biotite-bearing paragneiss & \\
\hline $01 R N 61 B$ & $48637 \overline{5}$ & 7105321 & Eagle & A1 & Biotite-garnet amphibolite & \\
\hline 01RN75A & 487649 & 7112236 & Eagle & A1 & Amphibolite & \\
\hline 01RN102B & 488186 & 7120555 & Eagle & A1 & Amphibolite & \\
\hline 01RN117A & 489816 & 7107373 & Eagle & A1 & Amphibolite & \\
\hline O1RN128A & 488775 & 7106615 & Eagle & A1 & Amphibolite & \\
\hline 01RN134B & 492248 & 7110365 & Eagle & A1 & Amphibolite & \\
\hline 01RN136A & 490928 & 7116038 & Eagle & A1 & Amphibolite & \\
\hline 01RN151A & 495272 & 7104279 & Eagle & A1 & Amphibole gneiss & \\
\hline
\end{tabular}


Tabie 3. Location and description of rock samples collected in the Eagle and Tanacross quadrangles for major-oxide, minor oxide, and trace element analyses.

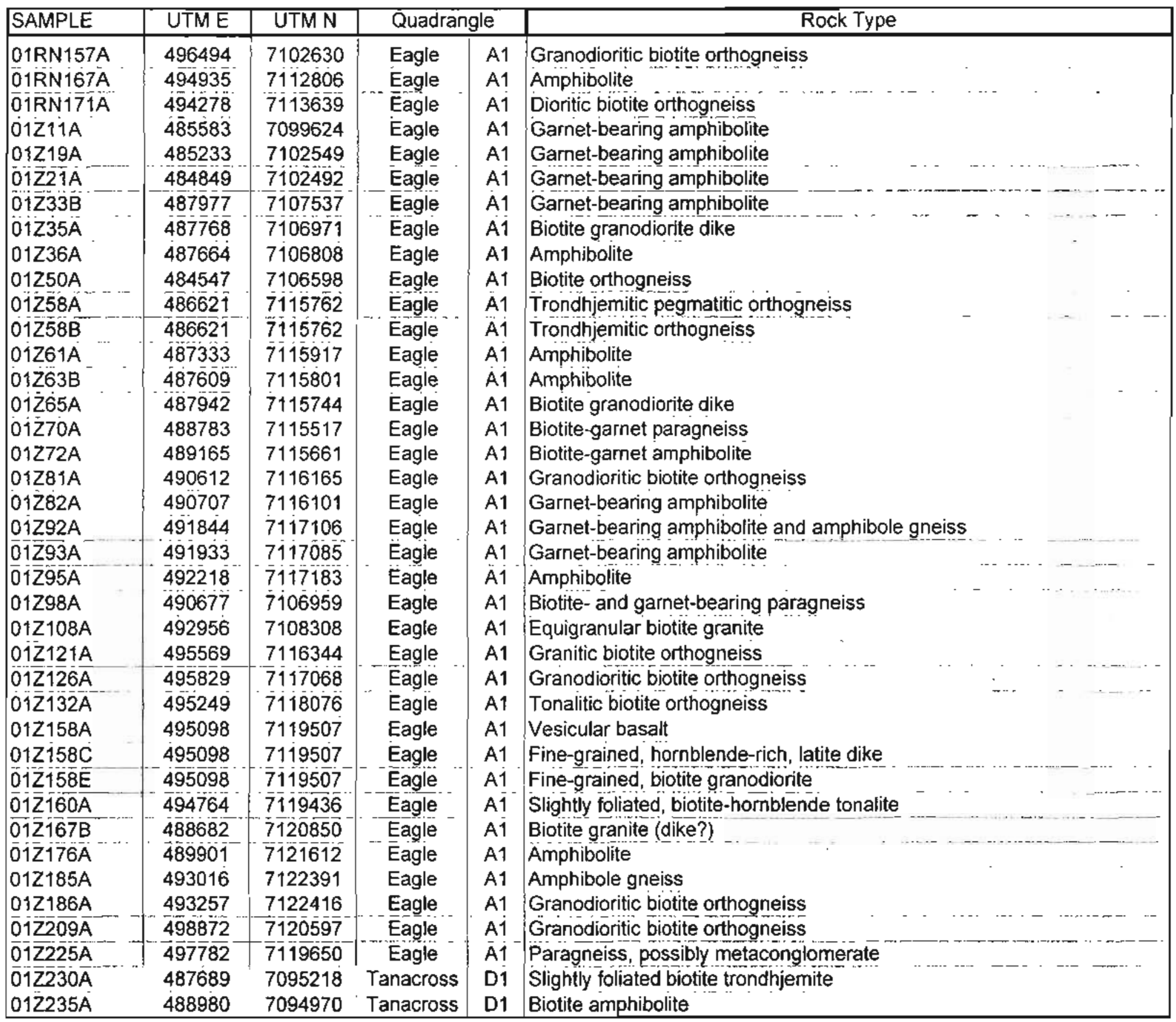


Table 3. Location and description of rock samples coliected in the Eagle and Tanacross quadrangles for major-oxide, minor oxide, and trace element analyses.

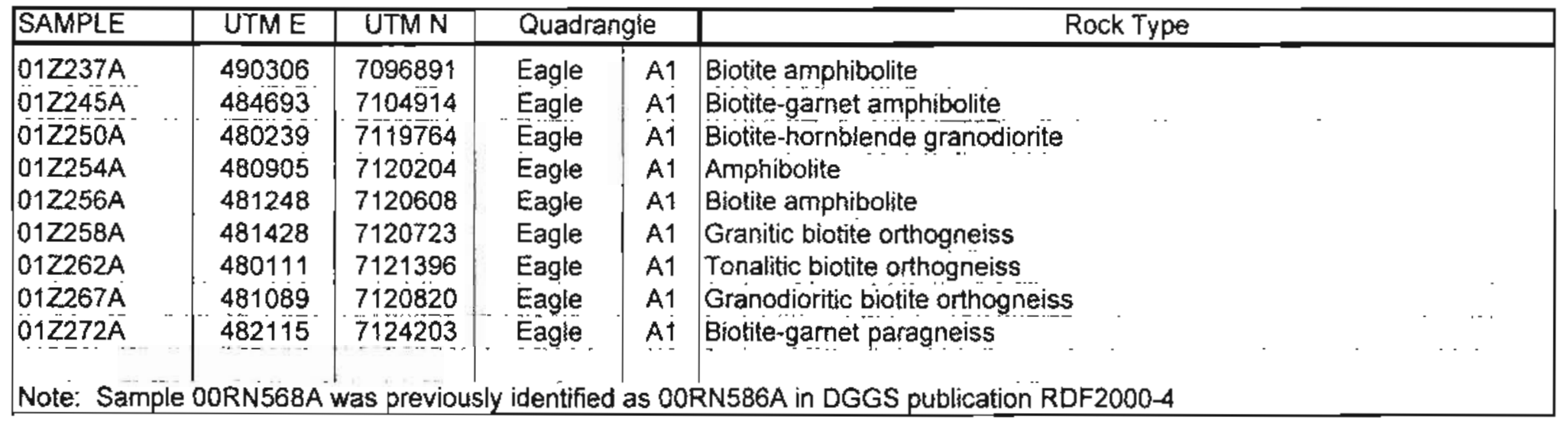


Table 4. Concentration of major-oxides, minor oxides, and trace elements in rock samples from the Eagle and Tanacross quadrangles, Alaska, Note: $-=$ not analyzed.

\begin{tabular}{|c|c|c|c|c|c|c|c|c|c|c|c|c|c|c|c|c|c|c|c|c|}
\hline $\begin{array}{l}\text { SAMPLE } \\
\text { NUMBER }\end{array}$ & $\begin{array}{l}\text { Al2O3 } \\
\%\end{array}$ & $\begin{array}{l}\mathrm{CaO} \\
\%\end{array}$ & $\begin{array}{l}\mathrm{Cr} 2 \mathrm{O3} \\
\%\end{array}$ & $\begin{array}{l}\text { Fe2O3 } \\
\%\end{array}$ & $\begin{array}{l}K 20 \\
\%\end{array}$ & $\begin{array}{l}\mathrm{MgO} \\
\%\end{array}$ & $\begin{array}{l}\mathrm{MnO} \\
\%\end{array}$ & $\mid \begin{array}{l}\mathrm{Na2O} \\
\%\end{array}$ & $\begin{array}{l}\mathrm{P} 2 \mathrm{O5} \\
\%\end{array}$ & $\begin{array}{l}\mathrm{SiO} 2 \\
\%\end{array}$ & $\begin{array}{l}\mathrm{TiO} 2 \\
\%\end{array}$ & $\begin{array}{l}\text { LOl } \\
\%\end{array}$ & $\begin{array}{l}\text { TOTAL } \\
\%\end{array}$ & $\begin{array}{l}\text { Ba } \\
\text { ppm }\end{array}$ & $\begin{array}{l}\mathrm{Rb} \\
\text { Ppm }\end{array}$ & $\begin{array}{l}\mathrm{Nb} \\
\mathrm{ppm}\end{array}$ & $\begin{array}{l}\text { Sr } \\
\text { ppm }\end{array}$ & \begin{tabular}{|l|}
$\mathrm{Zr}$ \\
$\mathrm{Ppm}$
\end{tabular} & \begin{tabular}{|l|}
$\mathrm{Y}$ \\
$\mathrm{ppm}$
\end{tabular} & $\begin{array}{c}\mathrm{BaO} \\
\%\end{array}$ \\
\hline 99MBW079 & 22.52 & 1.86 & 0.01 & 3.98 & 3.20 & 1.12 & 0.04 & 1.70 & 0.14 & 60.20 & 0.93 & 2.90 & 98.60 & 1485 & 138 & 26 & 280 & 204 & 44 & - \\
\hline W095B & 9.60 & 2.08 & $<.01$ & 1.71 & 1.11 & 0.73 & $<.01$ & 0.11 & 0.03 & 72.59 & 0.13 & 11.00 & 99.09 & 2460 & 78 & 34 & 2420 & 240 & 38 & $=$ \\
\hline 121 & 13.80 & 4.70 & $<.01$ & 5.60 & 0.75 & 2.78 & 0.09 & 3.74 & 0.11 & 65.92 & 0.59 & 0.95 & 99.03 & 250 & 22 & 10 & 198 & 87 & 30 & - \\
\hline $14-6 / 28$ & 16.34 & 5.37 & .01 & 7.36 & 2.10 & 5.04 & 0.13 & 3.20 & 0.19 & $\overline{5} 7.54$ & 0.73 & 1.06 & 99.12 & -- & 64 & 10 & $-\cdots$ & 129 & 22 & 0.03 \\
\hline $32-6 / 29$ & 14.71 & $9.7 \overline{7}$ & $<.01$ & 10.83 & 1.48 & 8.19 & 0.18 & 2.56 & 0.22 & 49.30 & 0.81 & 1.45 & 99.55 & - & 34 & 6 & -- & 62 & 20 & 0.02 \\
\hline $092-6 / 20$ & 18.51 & 4.10 & $<.01$ & 11.18 & 2.82 & 4.01 & 0.26 & 5.13 & 0.12 & 51.56 & 0.81 & 0.85 & 99.43 & $\overline{-}$ & 56 & 8 & -- & 54 & $1 \underline{8}$ & 0.08 \\
\hline $95-6 / 20$ & 17.26 & 6.31 & $<.01$ & $9 . \overline{5}$ & $\overline{2} . \overline{44}$ & 6.26 & 0.18 & 4.39 & 0.35 & 50.15 & 1.20 & 1.17 & 99.74 & $\overline{-}$ & $5 \overline{8}$ & 12 & - & 143 & $2 \overline{6}$ & 0.14 \\
\hline W194 & 14.52 & 1.43 & 01 & $\overline{0} . \overline{5} \overline{7}$ & $2.7 \overline{8}$ & 0.06 & 0.01 & 3.70 & 0.03 & 75.27 & 0.06 & 0.63 & 99.06 & $3 \overline{120}$ & $\overline{7} \overline{2}$ & 8 & 762 & 51 & & $\because$ \\
\hline$W 213 C^{-}$ & $14 . \overline{57}$ & 1.06 & 0.01 & 0.83 & 4.73 & 0.18 & 0.02 & 3.60 & 0.03 & 73.08 & $0 . \overline{08}$ & $0 . \overline{5} \overline{5}$ & 98.74 & 1995 & $8 \overline{0}$ & $\overline{6}$ & 554 & 69 & $1 \overline{4}$ & - \\
\hline W535 & $14 . \overline{47}$ & $3 . \overline{12}$ & 01 & 5.00 & 4.30 & 1.52 & 0.10 & 2.37 & 0.29 & $\overline{6} \overline{5} . \overline{5}$ & 0.56 & 1.52 & $9 \overline{8} .75$ & $1 \overline{75}$ & $\overline{8} 2$ & 18 & $65 \overline{4}$ & 162 & 26 & - \\
\hline$\sqrt{549}$ & .31 & 1.08 & 0.01 & .45 & 3.54 & 0.03 & 0.03 & 5.30 & 0.01 & 72.96 & 0.04 & 0.1 & 94 & 3570 & 64 & 10 & $96 \overline{8}$ & 48 & 6 & $\ldots$ \\
\hline & 13.68 & 1.80 & 0.03 & 6.75 & 3.18 & 4.18 & 0.05 & 1.3 & 0.23 & 62.54 & 0.92 & 3.99 & 59 & $\because-$ & & & & - & & - \\
\hline 134 & $13 . \overline{8} 5$ & $2.1 \overline{6}$ & 01 & 4.96 & 2.62 & 2.44 & 0.07 & 3.45 & 0.18 & $6 \overline{8} .09$ & 0.70 & 1.05 & $99.5 \overline{7}$ & 1885 & 104 & 16 & 264 & 171 & 32 & - \\
\hline 61 & 15.49 & 14.63 & 0.15 & 6.61 & 0.11 & 1.03 & 0.11 & 1.06 & $<.01$ & 46.97 & 0.25 & 2.59 & & - & - & & - & - & -- & $-\infty$ \\
\hline 61 & 79 & & 0.10 & 6.59 & 0.10 & & & & & & & & & 40 & 14 & 6 & 82 & 15 & 12 & -- \\
\hline $92 \mathrm{~A}$ & 19.99 & 12.05 & 0.05 & 4.87 & 0.60 & 9.37 & 0.08 & 1.77 & 0.03 & 46.43 & 0.12 & 3.52 & 98.88 & & & & & & & -- \\
\hline $92 \mathrm{~A}$ & 20.37 & 12.06 & 0.04 & 4.80 & 0.62 & 9.40 & 0.08 & 1.90 & 0.01 & 46.37 & 0.11 & 3.55 & 99.31 & 585 & 22 & 4 & 128 & 15 & 6 & $-\infty$ \\
\hline $12 \mathrm{~A}$ & 14.74 & 9.88 & 0.01 & 1.08 & 0.49 & 7.59 & 0.18 & 3.43 & 0.14 & 49.01 & 1.52 & 1.71 & 78 & 165 & 18 & 10 & 148 & 93 & 28 & $=-$ \\
\hline $3 B$ & 4.09 & 1.21 & 01 & 1.45 & & 0.24 & & 1.72 & & & & & & 2260 & 204 & 20 & 254 & 120 & 26 & -- \\
\hline & & 1.23 & & & & 2.83 & & & & & & & & 910 & 162 & 28 & 90 & 192 & 20 & $\cdots$ \\
\hline $26 \mathrm{~A}$ & 13.62 & 2.72 & $<.01$ & 5.08 & 3.30 & 1.81 & 0.07 & 1.00 & 0.09 & 68.54 & 0.62 & 1.97 & 98.82 & $\ldots-$ & $-\ldots$ & & & --- & 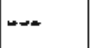 & $\because$ \\
\hline $2 \overline{6 A}$ & $\overline{7} \overline{4}$ & 2.77 & 01 & 5.30 & 3.14 & 1.75 & 0.07 & 1.05 & $0.1 \overrightarrow{0}$ & 68.01 & 0.65 & 200 & $9 \overline{8} . \overline{5} \ddot{8}$ & $9 \overline{9} \overline{5}$ & 112 & 20 & 164 & 231 & 30 & $-\infty$ \\
\hline $6 \mathrm{~B}$ & & 11.00 & 0.01 & 10.23 & & & .78 & 1.59 & 0.05 & & & & & 160 & 16 & 6 & 116 & 27 & 16 & $\rightarrow$ \\
\hline & 15 & 3.86 & $0 \dagger^{\cdots}$ & 7.22 & 0.09 & 8.46 & 0.14 & 1.92 & $<.01$ & 48.88 & 0.42 & 1.69 & $98.83-$ & & & & & & -- & - \\
\hline 34 & 5.27 & 13.71 & .01 & 7.25 & 0.10 & 8.33 & 0.14 & 1.89 & 0.04 & 49.17 & 0.42 & 1.73 & 99.05 & 60 & 10 & 4 & 98 & 18 & 14 & $\cdots$ \\
\hline & & 0.16 & 0.02 & 2.19 & & 1.87 & 0.03 & 0.14 & 0.05 & 76.74 & 0.20 & 1.75 & & $--{ }^{\prime}$ & & & $-\quad$ & --- & --- & --- \\
\hline $4 \mathrm{~A}$ & & 6.12 & $<.01$ & 11.16 & 2.29 & 4.85 & 0.18 & 4.46 & 0.36 & 49.53 & 1.04 & 1.39 & & 2360 & 64 & 6 & 310 & 108 & 26 & -- \\
\hline $201 \mathrm{~A}$ & $2 . \overline{9}$ & $2 . \overline{3}$ & 0.01 & 1.87 & 1.07 & $1.4 \overline{0}$ & 0.04 & 2.18 & $\leq .01$ & $7 \overline{5} . \overline{2} 7$ & 0.12 & 1.59 & 98.75 & $=$ & $\cdots$ & $\ldots$ & $\overline{---}$ & $-\infty$ & -- & - \\
\hline $236 \mathrm{~A}$ & $\overline{8} \overline{5}$ & 12,12 & 0.06 & 11.33 & 0.49 & 8.23 & 0.18 & 2.16 & 0.12 & $4 \overline{5} . \overline{9}$ & 1.22 & 2.26 & 99.01 & 355 & 20 & 6 & 220 & 81 & 22 & - \\
\hline $322 \mathrm{~A}$ & $2.7 \overline{5}$ & 14.72 & 0.03 & 7.66 & 0.07 & 11.60 & 0.14 & 1.15 & 0.22 & 48.69 & 0.40 & 1.68 & & 40 & 12 & $\overline{6}$ & 50 & 18 & 14 & --- \\
\hline & 17.44 & 0.69 & 01 & 1.96 & 0.09 & 0.53 & 0.03 & $\leq .01$ & 0.24 & 75.94 & 0.95 & 1.64 & $99.51=$ & --- & 8 & 28 & - & 210 & 22 & $<.01$ \\
\hline $345 \mathrm{~A}$ & 11.82 & $10 . \overline{54}$. & 0.10 & 7.53 & 0.54 & 12.85 & 0.15 & 1.72 & 0.14 & 50.10 & 0.20 & 3.58 & 99.27 & 610 & 24 & 6 & 102 & 18 & 14 & $-\infty$ \\
\hline $34 \overline{9}$ & 15.22 & 8.85 & $<01$ & $\overline{9} .92$ & 0.42 & 8.35 & 0.13 & $2 . \overline{9} 2$ & 0.10 & 50.34 & 0.85 & 1.93 & 99.03 & 795 & 18 & 8 & 324 & 57 & 20 & -- \\
\hline $349 B$ & 2.65 & 11.92 & 0.10 & 5.97 & 0.23 & 19.52 & 0.19 & 0.02 & $\underline{0.03}$ & 55.68 & 0.04 & 3.10 & 99.45 & 455 & 16 & 6 & & 12 & 0 & - \\
\hline $0 O R F 357 \bar{C}$ & 15.33 & $\overline{7}, t 2$ & 0.01 & 9.90 & 0.31 & 7.30 & 0.15 & 3.05 & 0.35 & 51.97 & 0.98 & 2.85 & 99.32 & 225 & 16 & 8 & 422 & 75 & 24 & - \\
\hline OORF 376 & 14.76 & 6.50 & $<.01$ & 12.20 & 0.54 & 5.42 & 0.20 & 4.21 & 0.11 & 52.08 & 1.25 & 1.29 & 98.56 & 195 & 20 & 8 & 144 & 66 & 24 & - \\
\hline OORF454 & 9.51 & 0.64 & 0.02 & $2.7 \overline{5}$ & 2.48 & $0.7 \overline{6}$ & 0.04 & 1.17 & 0.09 & $7 \overline{9} . \overline{3}$ & $0 . \overline{3} \overline{5}$ & 1.36 & 99.00 & 650 & 96 & 14 & 70 & 201 & 22 & - \\
\hline
\end{tabular}


Table 4. Concentration of major-oxides, minor oxides, and trace elements in rock samples from the Eagie and Tanacross quadrangles, Alaska. Note: $-=$ not analyzed.

\begin{tabular}{|c|c|c|c|c|c|c|c|c|c|c|c|c|c|c|c|c|c|c|c|c|}
\hline $\begin{array}{l}\text { SAMPLE } \\
\text { NUMBER }\end{array}$ & $\begin{array}{l}\mathrm{A} / 203 \\
\%\end{array}$ & $\frac{\mathrm{CaO}}{\%}$ & \begin{tabular}{|l|}
$\mathrm{Cr} 2 \mathrm{O} 3$ \\
$\%$
\end{tabular} & \begin{tabular}{|l|}
$\mathrm{Fe} 2 \mathrm{O} 3$ \\
$\%$
\end{tabular} & $\left|\begin{array}{l}\mathrm{K} 2 \mathrm{O} \\
\%\end{array}\right|$ & $\begin{array}{l}\mathrm{MgO} \\
\%\end{array}$ & $\begin{array}{l}\mathrm{MnO} \\
\%\end{array}$ & $\begin{array}{l}\mathrm{Na} 2 \mathrm{O} \\
\%\end{array}$ & $\begin{array}{l}\mathrm{P} 205 \\
\% \\
\%\end{array}$ & $\mid \begin{array}{l}\mathrm{SiO} 2 \\
\%\end{array}$ & $\begin{array}{l}\mathrm{TiO} 2 \\
\%\end{array}$ & $\begin{array}{l}\text { LOl } \\
\%\end{array}$ & $\begin{array}{l}\text { TOTAL } \\
\%\end{array}$ & $\begin{array}{l}\text { Ba } \\
\text { ppm }\end{array}$ & $\begin{array}{l}\text { Rb } \\
\text { ppm }\end{array}$ & $\begin{array}{l}\text { Nb } \\
\mathrm{ppm}\end{array}$ & $\begin{array}{l}\mathrm{Sr} \\
\mathrm{ppm}\end{array}$ & $\begin{array}{l}\mathrm{Zr} \\
\mathrm{ppm}\end{array}$ & $\begin{array}{l}Y \\
\text { ppm }\end{array}$ & $\%$ \\
\hline DORF467 & 4.77 & 10.60 & 0.01 & 11.85 & 0.61 & 5.05 & 0.19 & 2.87 & 0.17 & 50.06 & 1.62 & 1.37 & 99.17 & 165 & 28 & 10 & 156 & 96 & 26 & - \\
\hline & .07 & 1.36 & $<01$ & & $3 . \dot{69}$ & 0.50 & 0.13 & $<.01$ & 0.03 & $\overline{7} 1.03$ & 0.17 & 5.83 & 98.52 & $\ldots$ & - & - & 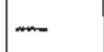 & - & - & -- \\
\hline & 5.16 & 0.17 & 0.01 & 0.50. & 1.65 & 0.52 & 0,01 & $<.01$ & 0.06 & 87.61 & 0.27 & 2.58 & 98.54 & 1645 & 62 & 12 & 120 & 66 & 16 & \\
\hline & 13.91 & 6.53 & 0.01 & 6.50 & 2.40 & 4.95 & 0.13 & 2.45 & 0.23 & 56.31 & 0.62 & 4.89 & 98.93 & 1165 & 66 & 12 & 472 & 108 & 22 & \\
\hline & 1.21 & 11.19 & 0.02 & 12.70 & 1.74 & 10.50 & 0.18 & 3 & 0.92 & 0.08 & 3.04 & 4.06 & 99.02 & 795 & 54 & 102 & 972 & 225 & 24 & - \\
\hline & 32 & 0.93 & 0.01 & 4.00 & 4.27 & 0.82 & 0.07 & 1.01 & 0.10 & 0.98 & 0.42 & 2.79 & 98.72 & - & - & $\ldots$ & $-\infty$ & - & $-\cdots$ & - \\
\hline & & 0.18 & $\overline{0.0 j}$ & 3.76 & 1.81 & 4.74 & 0.1 & 0.92 & 0.13 & & 0.46 & & $9 \ddot{9} .04$ & - & -- & - & - & -.. & & 更 \\
\hline & & 10.66 & 0.05 & 11.77 & 0.64 & 4.90 & & 3. & 0.22 & 706 & 1.88 & & 98.64 & 530 & 22 & 10 & 236 & 132 & 32 & - \\
\hline & & 8.57 & 0.04 & 11.44 & 16 & 5.45 & 0.20 & 2.92 & 0.19 & & 1.17 & & 8.86 & 39 & 32 & 10 & 258 & 78 & 24 & $\cdots$ \\
\hline & & 8.28 & 0.01 & 10.26 & 0.57 & 5.15 & 0.24 & 1.98 & 0.19 & 3.11 & 1.09 & & $8 . \ddot{42}$ & 230 & 18 & 8 & 206 & 63 & 24 & $-\cdots$ \\
\hline & 16.18 & 10.18 & $<.01$ & 8.93 & 1.66 & 6.99 & 0.17 & 2.68 & 0.23 & 9.07 & 1.43 & 1.43 & 8.95 & 905 & 48 & 24 & 476 & 108 & 22 & - \\
\hline & 19 & 10.79 & & 13.15 & 1.10 & 4.18 & 0.18 & 3.19 & 0.45 & & 3.03 & & .54 & 40 & 20 & 38 & 588 & 132 & 18 & - \\
\hline & .51 & 6.50 & 01 & 8.51 & 2.68 & 3.95 & 0.17 & 4.18 & 0.20 & 4.15 & 0.78 & & 9.06 & 1560 & 54 & 14 & 574 & 7 & 18 & 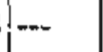 \\
\hline & 14.88 & 10.79 & 0.01 & 11.93 & 1.51 & 7.71 & 0.18 & 2.26 & 0.53 & 4.74 & 1.99 & & 8.58 & 490 & 34 & 34 & 504 & 180 & 26 & -- \\
\hline & 1500 & 8.15 & $<01$ & 10.42 & 0.97 & 6.52 & 0.18 & 4.08 & 0.30 & 1.17 & 1.06 & 1.28 & 99.14 & $=$ & 26 & 7 & - & 65 & 22 & $<.01$ \\
\hline & $17.9 \overline{5}$ & 7.20 & 01 & 9.84 & $\overline{0} . \overline{5} 2$ & 5.50 & 0.10 & 2.56 & 0.09 & 52.96 & 0.68 & 1.35 & 98.75 & 220 & 18 & 8 & 33 & 54 & 20 & -- \\
\hline & .12 & 8.31 & 01 & 10.78 & 38 & 5.51 & 0.17 & 2.28 & 0.08 & 1.86 & 0.69 & & & 21 & 14 & 8 & 236 & 54 & 18 & - \\
\hline & 60 & 9.05 & 0.01 & .39 & 1.11 & 7.63 & 0.28 & 2.88 & 0.14 & 8.02 & 0.93 & & 3.70 & 290 & 36 & 12 & 266 & 60 & 18 & -- \\
\hline & 15.82 & 6.20 & 0.03 & 9.63 & 0.89 & 4.98 & 0.16 & 5.08 & 0.08 & 3.89 & 0.77 & & 8.62 & 82 & 26 & 6 & 166 & 48 & 18 & - \\
\hline & 17.04 & 7.76 & $<.01$ & 10.38 & 1.74 & 6.77 & 0.30 & 3.93 & 0.26 & 48.32 & 0.88 & 27 & 98.76 & - & 44 & 9 & $\sim$ & 67 & 22 & no \\
\hline & .47 & 9.52 & & & & 8.49 & & & 0.26 & 7.39 & 21 & & & - & $7 \mathrm{a}$ & 6 & - & 01 & 24 & \\
\hline & & 11.20 & & 17.60 & & & & 1.73 & 0.36 & & 65 & & 9.71 & ב-ב & 22 & 6 & - & 41 & 24 & 0.0 \\
\hline & & 8.03 & & & 0.81 & & & 2.42 & 0.22 & & 44 & & & & 22 & $<10$ & - & 110 & 34 & 0.0 \\
\hline & & & & & & & & & 0.27 & & & & & & 16 & $\mid<10$ & $\cdots$ & & 28 & 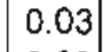 \\
\hline & & 10.60 & & & & & & & & & & & & & 20 & $<10$ & $\cdots$ & 90 & 30 & \\
\hline & & & & & & & & & & & $4 \overline{4}$ & & & & 20 & $<10$ & - & 50 & 16 & 0.0 \\
\hline & & 10.20 & & & & 5.82 & & & & & & & & & 18 & 10 & - & & 22 & 0.0 \\
\hline & & & & & & 0.29 & 0.01 & & 05 & & & & .29 & 2370 & 46 & $\mid<10$ & $-\infty$ & 80 & 12 & 0.2 \\
\hline & & & & & & & & & & & 0.79 & & & & 18 & $<10$ & & & & \\
\hline & & & 0.01 & & & & & & & & & & & & 44 & $\leq 10$ & & 80 & 22 & 0.1 \\
\hline & & & & & 2. & & & & & & & & & 1480 & 80 & 20 & & 190 & 26 & 0.1 \\
\hline & & & $<01$ & 11.98 & & 5.8 & & & & 51.64 & 0.91 & & & 28 & 16 & $<10$ & & 50 & 20 & $<.0^{\circ}$ \\
\hline & & 4.64 & $<.01$ & & & 4.1 & & & 0.07 & 64.47 & 0.43 & & 99.37 & 1280 & 74 & 10 & -- & 120 & 28 & 0.1 \\
\hline & & 12.21 & & 10. & & 7.7 & & & 0.50 & 46.38 & 2.16 & & & & 20 & 70 & - & 180 & 26 & 0.0 \\
\hline & & 9.24 & 0.03 & 10.0 & & 6.21 & & & 0.22 & 49.75 & 1.77 & & 99.96 & & 32 & 20 & $\because$ & 140 & 30 & 0.0 \\
\hline & & 1.70 & $<.01^{\circ}$ & & 3.06 & 0.20 & $\ddot{0 . C}$ & & 03 & $6 \overline{9} . \overline{9}$ & 0.11 & & 99.02 & 2090 & 68 & $<10$ & 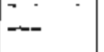 & 00 & 10 & 0.24 \\
\hline T.80 & 4.80 & 1.36 & 0.02 & .19 & 3.08 & 0.32 & 0.04 & 4.76 & 0.03 & 72.83 & 0.12 & 0.54 & 99.52 & 2580 & 52 & $<10$ & 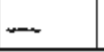 & 80 & 12 & 0.28 \\
\hline
\end{tabular}


Table 4. Concentration of major-oxides, minor oxides, and trace elements in rock samples from the Eagle and Tanacross quadrangles, Alaska.

Note: $-=$ not analyzed.

\begin{tabular}{|c|c|c|c|c|c|c|c|c|c|c|c|c|c|c|c|c|c|c|c|c|}
\hline $\begin{array}{l}\text { SAMPLE } \\
\text { NÜMBER }\end{array}$ & $\begin{array}{l}\text { Al2O3 } \\
\%\end{array}$ & $\begin{array}{l}\mathrm{CaO} \\
\%\end{array}$ & $\begin{array}{l}\mathrm{Cr} 2 \mathrm{O} 3 \\
\%\end{array}$ & $\begin{array}{l}\mathrm{Fe} 2 \mathrm{O} 3 \\
\%\end{array}$ & $\begin{array}{l}\mathrm{K} 2 \mathrm{O} \\
\%\end{array}$ & $\begin{array}{l}\mathrm{MgO} \\
\%\end{array}$ & $\begin{array}{l}\mathrm{MnO} \\
\%\end{array}$ & $\mid \begin{array}{l}N a 20 \\
\%\end{array}$ & $\begin{array}{l}\mathrm{P} 2 \mathrm{O5} \\
\%\end{array}$ & $\frac{\mathrm{SiO} 2}{\%}$ & $\begin{array}{l}\mathrm{TiO2} \\
\%\end{array}$ & $\begin{array}{l}\mathrm{LOI} \\
\%\end{array}$ & $\begin{array}{l}\text { TOTAL } \\
\% \%\end{array}$ & $\begin{array}{l}\text { Ba } \\
\ddot{\rho p m} \\
\text { ppm }\end{array}$ & $\begin{array}{l}\mathrm{Rb} \\
\mathrm{ppm}\end{array}$ & $\begin{array}{l}\mathrm{Nb} \\
\mathrm{ppm}\end{array}$ & $\begin{array}{l}\text { Sг } \\
\text { ppm }\end{array}$ & \begin{tabular}{|l}
$\mathrm{Zr}$ \\
$\mathrm{ppm}$
\end{tabular} & $\begin{array}{l}Y \\
\text { ppm }\end{array}$ & $\begin{array}{c}\mathrm{BaO} \\
\%\end{array}$ \\
\hline $01 \mathrm{KC} 16 \mathrm{~B}$ & 14.81 & 1.62 & $<.01$ & 1.18 & 2.21 & 0.23 & 0.03 & 5.29 & 0.03 & 73.48 & 0.16 & 0.39 & 99.80 & 2110 & 46 & $<10$ & - & 80 & 14 & \\
\hline & .39 & 1.16 & 01 & 0.82 & 1.67 & 0.25 & 0.02 & $\overline{7} . \overline{3}$ & 0.03 & 71.14 & $0.0 \overline{9}$ & 0.31 & 99.24 & & 28 & 30 & --- & 90 & 10 & 0.12 \\
\hline & 7.18 & 1.76 & 01 & 3.56 & 0.40 & 0.08 & 0.19 & 8.19 & 0.08 & $66 . \overline{4} 4$ & 0.34 & & 98.64 & & 10 & 240 & & 930 & 46 & $<.01$ \\
\hline $9 \mathrm{~B}$ & 13.41 & 11.83 & 01 & 16.80 & 1.11 & 5.64 & 0.22 & 2.29 & 0.61 & 41.34 & 4.49 & 1.19 & 99.04 & 250 & 18 & 20 & & 90 & 18 & 0.03 \\
\hline $41 \mathrm{~A}$ & 14.07 & 1.54 & .01 & 2.08 & 2.17 & 0.63 & 0.05 & 4.23 & 0.05 & 73.46 & 0.29 & 0.75 & 99.67 & 2200 & 50 & 10 & & 130 & 14 & 0.24 \\
\hline $42 \mathrm{~A}$ & 14.14 & $2 . \overline{12}$ & .01 & 2.01 & 2.70 & 0.44 & 0.05 & 4.00 & 0.06 & 71.15 & $0 . \overline{2}$ & 0.60 & $98 . \overline{0} \overline{7}$ & 2900 & 50 & 10 & |--- & 150 & 28 & 0.33 \\
\hline $47 \mathrm{~B}$ & 13.65 & 3.30 & 0.0 & 4.86 & 1.41 & & 0 . & 92 & 0.0 & 68.67 & 0.40 & 0.58 & 99.41 & 650 & 42 & 10 & - & 100 & 24 & 0.05 \\
\hline$\overline{1} 1 \overline{\mathrm{B}}$ & 14.72 & $\overline{2} . \overline{6} \overline{9}$ & $\overline{01}^{-}$ & $\overline{1} . \overline{0} \overline{2}$ & 1.55 & 0.22 & 0.01 & $4 . \overline{6} 9$ & 0.03 & 72.51 & 0.14 & 0.29 & $9 \ddot{8} .19$ & 1630 & 34 & $<10$ & - & 80 & 12 & 0.18 \\
\hline $7 \mathrm{~A}$ & $1 \overline{7} .26$ & 15.09 & 01 & 15.12 & 0.59 & 2.76 & 0.20 & .45 & $0.2 \overline{3}$ & 43.74 & 1.42 & 1.42 & 99. & 150 & 20 & 10 & - & 70 & 24 & $<.01$ \\
\hline & & $\overline{7} . \overline{7} \overline{5}$ & $01 "$ & 11.16 & 2.97 & 5.00 & 0.17 & 12 & 0.40 & & 1.10 & & 98. & & $\overline{6} 6$ & 10 & & 120 & 26 & 0.12 \\
\hline & 82 & 5.98 & 01 & 7.14 & 2.99 & 2.70 & 0.1 & 2.91 & 0.29 & 59.35 & 0.70 & 1.12 & 99.46 & 2000 & 74 & 10 & - & 100 & 24 & 0.21 \\
\hline $1 \mathrm{~A}$ & 14.22 & $9 . \overline{8} 2$ & 01 & 14.81 & 1.71 & 6.68 & 0.21 &.$\overline{91}$ & 0.77 & $5.9 \overline{5}$ & 1.26 & 1.74 & 9923 & $820^{\circ}$ & 38 & 10 & - & 60 & 24 & 0.10 \\
\hline & 44 & 7.67 & 0.01 & 10.40 & 2.31 & 4.15 & 0.1 & 31 & 0.46 & 3.96 & 0.91 & 1.44 & 99.50 & 1920 & 56 & 10 & -- & 00 & 2 & 0.20 \\
\hline & & $\overline{7} .29$ & $.01^{--}$ & 5.66 & 2.06 & 2.03 & & & 0.24 & 8.43 & 0.61 & 1.38 & & & 50 & 10 & & & 0 & \\
\hline & 6.29 & 1.57 & & 0.97 & & & & & & 68. & $0 . \overline{1}$ & 0.24 & 8 & 2860 & 80 & 20 & & 90 & 0 & 0.34 \\
\hline $5 \mathrm{D}$ & 11.21 & 11.84 & 01 & 21.41 & 2.29 & 9.60 & 0.19 & 0.83 & 1.80 & 34.91 & 1.52 & 3.91 & 99.57 & 390 & 54 & $<10$ & $=\ldots$ & 30 & 18 & 0.03 \\
\hline N1OA & 16.64 & 1.58 & 01 & 0.82 & $\overline{1} .61$ & 0.15 & 0.01 & .29 & 0.03 & 69.68 & 0.09 & 0.64 & 07 & 3060 & 20 & $1 \overline{0}$ & - & 70 & $\overline{8}$ & \\
\hline & & 5.40 & & & & & & & & & & & & & 40 & 10 & & 100 & 20 & 0.05 \\
\hline $150 \vec{A}$ & 2.07 & 1.18 & $<.01$ & 2.17 & 1.15 & 0.10 & 0.02 & 4.85 & 0.04 & $\overline{7} \overline{5} . \overline{9} 1$ & $0 . \overline{2} \overline{7}$ & 0.45 & 98.21 & 270 & 32 & 10 & $=$ & 170 & 34 & .01 \\
\hline N52A & $1 \overline{4} \cdot \overline{3}$ & 14.60 & 01 & 10.95 & 0.21 & 6.37 & $\overline{0} .11$ & $1 \overline{9}$ & $0 . \overline{08}$ & $46.9 \overline{3}$ & $0 . \overline{3} \overline{6}$ & 1.28 & & 60 & 14 & 10 & - & 30 & 14 & $\overline{0} 1$ \\
\hline$\sqrt{57} \mathrm{~B}$ & 3.26 & .57 & & & 89 & & & & & & & & & $\overline{49} 0$ & 22 & 10 & & 120 & 2 & 0.05 \\
\hline & & 2.37 & $<.01$ & & & & & & & & & & 98.59 & 1970 & 52 & 10 & & 80 & 10 & 0.22 \\
\hline & 11.76 & 8.51 & 0.02 & 9.04 & 0.90 & 9.73 & $0+1$ & 10 & 0.10 & 55.05 & 0.35 & 1.18 & 99.01 & 430 & 22 & $<10$ & -- & 40 & 16 & 0.03 \\
\hline$\sqrt{73 \mathrm{~A}}$ & 14.51 & 8.79 & 0.02 & 11.30 & 0.93 & 6.57 & 04 & 81 & 0.43 & 45.99 & 2.08 & 5.87 & 99.56 & 500 & 30 & 20 & - & 200 & 34 & .05 \\
\hline$\sqrt{7} \overline{7} \mathrm{~B}$ & $1 \overline{5} . \overline{4} 6$ & 2.27 & $<.01$ & 1.26 & 46 & 0.41 & & & & & 16 & & & & 58 & $<10^{\circ}$ & & 110 & 10 & 0.22 \\
\hline & & $\overline{2} . \overline{5} 2$ & & 3.81 & 99 & & & & & & 0.52 & & & & 68 & 60 & & 260 & 26 & 0.28 \\
\hline & & 2.17 & $<.01$ & 1.44 & 0.84 & 0.30 & & & 0.03 & 72.44 & 0.16 & 0.60 & 98.25 & 2990 & 22 & 10 & $\rightarrow$ & 80 & 16 & 0.35 \\
\hline 11920 & 5.58 & 3.05 & $<.01$ & 1.30 & 2.12 & 0.48 & 0.0 & 435 & 0.06 & 71.38 & 0.17 & 0.28 & & 1940 & 48 & 10 & & 110 & 8 & 0.23 \\
\hline & 15.84 & 1.68 & $<.01$ & 0.94 & 2.01 & 0.17 & $0 . \bar{c}$ & 6.20 & 0.03 & & 0.10 & & & 1770 & 38 & $<10$ & $=$ & 70 & - & 0.20 \\
\hline & $\overline{9} . \overline{7} \overline{2}$ & 5.21 & $<.01$ & 1.94 & $1 . \overline{29}$ & 0.96 & & & & & & & & 380 & $5 \overline{2}$ & 10 & & 250 & 22 & 0.01 \\
\hline & $13.1 \overline{1}$ & 5.38 & 0.03 & $4.4 \overline{7}$ & 0.75 & 1.77 & & & & 69.47 & & $\overline{0} . \overline{8}$ & & $3 \overline{7}$ & 16 & 10 & $-\cdots$ & 80 & 22 & 0.03 \\
\hline & & 0.51 & $<.01$ & 0.37 & 3.82 & $<.01$ & & & 0.04 & $71 . \overline{32}$ & 0.04 & 0.47 & & 2440 & 62 & $<10$ & -- & 70 & 8 & 0.30 \\
\hline & 14.24 & 1.05 & $<.01$ & 1.38 & 3.75 & 0.23 & & & 0.06 & 72.30 & 0.19 & 0.79 & 98.64 & 2000 & $B 2$ & 10 & --- & 130 & 14 & 0.23 \\
\hline (1) & 14.73 & 2.75 & & 2.88 & 3.73 & & & & & 69.44 & & 0.85 & 98.63 & 1740 & 84 & 20 & - & 100 & 16 & 0.19 \\
\hline$M 174$ & 14.45 & 9.40 & 0.10 & 10.60 & 0.36 & 10.02 & & 1.77 & 0.23 & 50.30 & 0.55 & 1.46 & 99.41 & 70 & 14 & 10 & $=$ & 50 & 20 & $<.01$ \\
\hline AUI & & 1.15 & $<.01$ & $0.5 \overline{0}$ & 0.50 & $0 . \overline{0} \overline{9}$ & 0.01 & 7.84 & $<01$ & 72.69 & $0 . \overline{0}$ & $0.2 \overline{8}$ & 98.68 & 800 & 12 & & $-\infty$ & $\overline{7} \overline{0}$ & 0 & 0.07 \\
\hline 01MBW183A & 15.60 & 1.39 & $<.01$ & 0.77 & 1.88 & 0.03 & 0.01 & 4.77 & 0.01 & 74.25 & 0.08 & 0.68 & 99.93 & $2 \overline{9} 10$ & 36 & $<10$ & $\cdots$ & 100 & 14 & 0.32 \\
\hline
\end{tabular}


Table 4. Concentration of major-oxides, minor oxides, and trace elements in rock samples from the Eagle and Tanacross quadrangles, Alaska. Note: -- = not analyzed.

\begin{tabular}{|c|c|c|c|c|c|c|c|c|c|c|c|c|c|c|c|c|c|c|c|c|}
\hline $\begin{array}{l}\text { SAMPLE } \\
\text { NUMBER }\end{array}$ & $\begin{array}{l}\mathrm{Al} 2 \mathrm{O} 3 \\
\%\end{array}$ & $\begin{array}{l}\mathrm{CaO} \\
\%\end{array}$ & $\begin{array}{l}\mathrm{Cr} 2 \mathrm{O} 3 \\
\%\end{array}$ & {$\left[\begin{array}{l}F e 203 \\
\%\end{array}\right.$} & $\begin{array}{l}\mathrm{K} 2 \mathrm{O} \\
\%\end{array}$ & $\begin{array}{l}\mathrm{MgO} \\
\%\end{array}$ & $\begin{array}{l}\text { MnO } \\
\%\end{array}$ & $\begin{array}{l}\mathrm{Na} 2 \mathrm{O} \\
\%\end{array}$ & $\begin{array}{l}\mathrm{P} 2 \mathrm{O} 5 \\
\%\end{array}$ & $\begin{array}{l}\mathrm{SiO} 2 \\
\%\end{array}$ & $\begin{array}{l}\text { TiO2 } \\
\%\end{array}$ & $\begin{array}{l}\mathrm{LOI} \\
\%\end{array}$ & $\mid \begin{array}{l}\text { TOTAL } \\
\%\end{array}$ & $\begin{array}{l}\mathrm{Ba} \\
\mathrm{ppm}\end{array}$ & $\begin{array}{l}R b \\
p p m\end{array}$ & $\begin{array}{l}\mathrm{Nb} \\
\text { ppm }\end{array}$ & $\begin{array}{l}\mathrm{Sr} \\
\text { ppm }\end{array}$ & $\begin{array}{l}\mathrm{Zr} \\
\mathrm{ppm}\end{array}$ & $\begin{array}{l}Y \\
p p m\end{array}$ & $\begin{array}{c}\mathrm{BaO} \\
\%\end{array}$ \\
\hline iAde' & 14.02 & 8.07 & $<.01$ & 13.20 & 0.63 & 4.10 & 0.23 & 3.11 & 0.22 & 52.59 & 1.84 & 0.44 & 98.52 & 480 & 18 & $<10$ & --- & 110 & 36 & 0.04 \\
\hline $\mathrm{W} 225 \mathrm{~A}$ & $\overline{5} \overline{9}$ & 5.10 & $<.01$ & 6.48 & 2.23 & 4.07 & 0.11 & 2.08 & 0.09 & 64.26 & 0.44 & 1.18 & $9 \overline{9} .75$ & 990 & 72 & 10 & - & 100 & 24 & 0.10 \\
\hline$\sqrt{2} 26 \bar{B}$ & 2.46 & 11,00 & $\overline{0.02}$ & 6.19 & $0.60^{\circ}$ & 5.40 & 0.11 & $\overline{2} .14$ & 0.12 & $57 . \overline{54}$ & 0.77 & 2.32 & $9 \ddot{8} \overline{7} \overline{0}$ & 210 & 20 & $<10$ & $=$ & 60 & 20 & $<.01$ \\
\hline$\sqrt{244 A}$ & 13.77 & 1.28 & .01 & 2.40 & 2.84 & 0.52 & 0.04 & 4.15 & 0.07 & 73.18 & 0.25 & 0.76 & 99.34 & 800 & 68 & 10 & -- & 140 & 22 & 0.07 \\
\hline W245A & 16.29 & 3.53 & $<.01$ & 2.45 & 2.04 & 0.75 & 0.04 & 4.18 & 0.22 & 67.95 & 0.29 & 1.30 & 99.31 & 1710 & 46 & $10^{\circ}$ & - & 100 & 10 & 0.17 \\
\hline$W 246 B$ & 14.77 & 9.45 & $<.01$ & 12.59 & 0.55 & 5.44 & 0.28 & 2.29 & 0.36 & 49.67 & 1.91 & 1.80 & $99.1 \dot{5}$ & 250 & 18 & $<10$ & - & 80 & 28 & 0.01 \\
\hline $252 \mathrm{~A}$ & 16.25 & 6.12 & $<.01$ & $\overline{7} .67$ & 2.99 & 2.91 & 0.15 & 44 & 0.37 & 57.30 & 0.67 & 1.04 & 99.28 & 2160 & 64 & 10 & --- & 90 & 24 & 1.25 \\
\hline$\sqrt{255 \mathrm{~A}}$ & & 4.70 & $<.01$ & 6.02 & 3.71 & 1.59 & & & 0.36 & & 0.66 & 1.11 & 99.31 & 1440 & 98 & 20 & --- & 170 & 30 & 16 \\
\hline N287B & 16.45 & 7.67 & $<, 01$ & 8.68 & 0.41 & 5.24 & 0.17 & 3.99 & 0.09 & 53.94 & 1.00 & 1.42 & 99.15 & 500 & 18 & $<10$ & -- & 70 & 22 & 0.04 \\
\hline $288 \mathrm{~A}$ & 16.23 & 1.50 & $<.01$ & 0.50 & 0.83 & 0.10 & 0.01 & .71 & 0.02 & 73.89 & 0.05 & 0.56 & 99.71 & 1030 & 20 & 10 & & 50 & & .12 \\
\hline & & 6.79 & & 11.89 & 0.71 & 4.31 & 0.19 & & & & 1.22 & & & 780 & 20 & $<10^{\circ}$ & - & 60 & 26 & 1.07 \\
\hline$\sqrt{294 A}$ & 16.31 & 0.97 & .01 & 1.10 & 1.54 & 0.34 & 0.01 & 3.97 & 0.03 & 73.93 & 0.14 & 0.92 & 99.64 & 2340 & 30 & 10 & -- & 80 & 16 & 0.25 \\
\hline $304 \mathrm{~A}$ & 14.61 & 8.86 & $<.01$ & 13.17 & 0.53 & 5.13 & 0.23 & 3.33 & 0.20 & 49.51 & 1.71 & 1.87 & 99.17 & 190 & 20 & $<10$ & --- & 100 & 32 & $<.01$ \\
\hline & 14.26 & 4.30 & .01 & 4.07 & 1.60 & 0.84 & 0.07 & 96 & 0.07 & 69.92 & 0.46 & 0.97 & 99.63 & 730 & 40 & 20 & & 130 & 28 & \\
\hline & 14.59 & 10.52 & & 11.48 & 0.45 & & & & & & & & & NA & & 7 & & 95 & 32 & $<.01$ \\
\hline $319 \mathrm{~A}$ & 19.54 & $\ddot{6} . \overline{9} \overline{0}$ & $<.01$ & $8.2 \bar{B}$ & 0.28 & 4.50 & 0.11 & 5.32 & 0.07 & 52.69 & 0.54 & 1.52 & $9 \overline{9}, 7 \overline{6}$ & 80 & 14 & 10 & & 60 & 18 & $<.01$ \\
\hline $321 \mathrm{~A}$ & 1283 & 0.33 & $<. \overline{0} \overline{1}$ & 1.09 & 4.85 & $<.01$ & 0.05 & 3.62 & 0.01 & 74.29 & 0.09 & 0.73 & 98.02 & 9000 & & 20 & & 70 & 26 & 0.10 \\
\hline & & 0.44 & & & & & & & & & & & & 50 & & 20 & & 30 & 28 & \\
\hline$A$ & 14.58 & 4.48 & $<01$ & 7.87 & 1.75 & 3.03 & 0.27 & 1.65 & 0.12 & 63.16 & 0.66 & $1 . \overline{7} 7$ & 99.47 & 1060 & iR & 10 & & 120 & 26 & 0.10 \\
\hline $335 \mathrm{~A}$ & 14.17 & 6.49 & 0.01 & в. 07 & 1.44 & $5.4 \ddot{4}$ & 0.14 & j. 81 & $\dot{0} .09$ & 59.54 & 0.54 & 1.68 & 99.50 & 600 & & 10 & & 80 & 22 & .05 \\
\hline & & & & & & & & & & & & & & & & 10 & & 100 & 26 & \\
\hline & 17.85 & 7.29 & 0.01 & 11.52 & 0.48 & 3.48 & 0.38 & & 0.49 & 51.05 & 1.62 & 0.86 & & 290 & & 20 & & 190 & 34 & $<.01$ \\
\hline $367 \mathrm{~A}$ & $15 \overline{2} \overline{1}$ & $1 . \overline{3} \overline{8}$ & 01 & 0.79 & $2.4 \overline{5}$ & 0.14 & 0.01 & $\overline{5} .35$ & $0.0 \overline{3}$ & 71.47 & $\overline{0} . \overline{1}$ & 0.53 & $\overline{9} \overline{8} .03$ & $3 \overline{340}$ & 44 & $<10^{-}$ & - & 50 & 10 & 0.42 \\
\hline & 5.23 & 1.15 & .01 & 0.56 & 1.37 & 0.01 & .01 & 7.09 & $<.01$ & 71.65 & 0.07 & 0.42 & 98.08 & 2340 & 22 & $<10$ & & 60 & 0 & 0.31 \\
\hline & 5.96 & 4.34 & & 4.75 & 3.62 & 1.19 & & & & & & & & 1640 & & 20 & & & 26 & \\
\hline & 15.06 & 3.87 & .01 & 4.94 & 3.66 & 1.37 & 0.13 & 2.97 & 0.26 & 65.14 & 0.52 & 0.93 & 99.16 & 1800 & & 20 & -- & 160 & 28 & 0.20 \\
\hline 1381B & 15.58 & 3.74 & .01 & 2.22 & 2.56 & 0.71 & 0.04 & 4.64 & 0.11 & & 0.25 & & & 1670 & 8 & 10 & $\cdots$ & 110 & 10 & .19 \\
\hline & 15.55 & 1.34 & .01 & 0.48 & 1.69 & 0.03 & 0.01 & 6.20 & 0.03 & 72.26 & 0.04 & 0.44 & 98.16 & 650 & 50 & 10 & - & 50 & & 0.06 \\
\hline & 13.76 & 6.52 & & 14.26 & 0.46 & 3.60 & 0.22 & & 0.06 & 55.50 & 0.82 & & & 130 & 14 & & & 40 & 18 & $<.01$ \\
\hline & 15.35 & 3.29 & $<.01$ & 1.08 & 1.64 & 0.07 & 0.02 & & & 71.15 & & & & 940 & & $<10$ & - & & & 0.10 \\
\hline & & 2.72 & $<.01$ & & 1.62 & 0.29 & 0.02 & 4.85 & & & 0.16 & 0.62 & 98.55 & 1210 & 40 & $1<10$ & & 90 & 14 & 0.12 \\
\hline$\sqrt{4} 32 \mathrm{~A}$ & 16.12 & 6.63 & 0.01 & $6.7 \overline{2}$ & 0.53 & 3.35 & 0.14 & 4.43 & 0.15 & 59.12 & 0.86 & 1.01 & 99.13 & 410 & 12 & 10 & & 100 & 30 & 0.03 \\
\hline & $1 \overline{5} .0 \overline{0}$ & 5.91 & 0.01 & $\overline{6} .59$ & 0.99 & 4.54 & 0.19 & 2.70 & 0.11 & 59.78 & 0.70 & $1 . \overline{7} \overline{7}$ & 98.35 & -470 & $2 \overline{4}$ & $1 \overline{0}$ & & 120 & 28 & $\underline{0.03}$ \\
\hline$\sqrt{4} \overline{3} \overline{\mathrm{A}}$ & $2 \overline{0} \cdot \overline{6} \overline{0}$ & 0.17 & $<.01$ & $0 . \overline{8} 8$ & 2.64 & 0.34 & 0.01 & & 0.01 & & & $5 . \overline{5} \overline{9}$ & & 1670 & $\overline{70}$ & 10 & & 70 & 8 & 0.19 \\
\hline & 14.06 & 8.71 & 0.07 & 8.82 & 1.67 & 8.83 & 0.14 & & 0.27 & 52.00 & 1.19 & 1.81 & 99.60 & 870 & & 20 & & 120 & 22 & 0.08 \\
\hline & 15.26 & 5.46 & $<.01$ & 7.63 & 1.41 & 3.41 & 0.14 & 3.84 & 0.24 & 60.16 & 0.73 & 1.07 & 99.58 & 1610 & 36 & 20 & $=$ & 120 & 24 & 0.16 \\
\hline $01 \mathrm{RN} 46 \mathrm{~A}$ & $\overline{9} . \overline{2} \overline{7}$ & 1.47 & $0 . \overline{01}$ & 5.00 & 2.10 & 2.80 & 0.12 & 0.82 & $0 . \overline{1} 4$ & $7 \overline{5.68}$ & $\overline{0} . \overline{5} \overline{5}$ & 1.41 & $\overline{9} . \overline{5}$ & 1620 & 62 & 10 & - & 110 & 28 & 0.15 \\
\hline
\end{tabular}




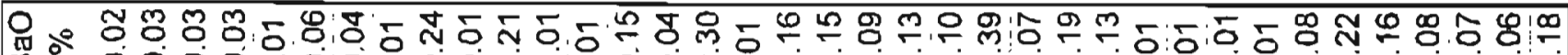

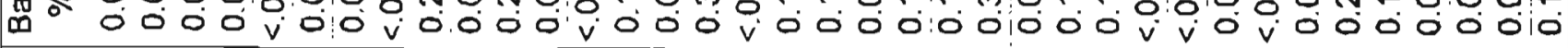

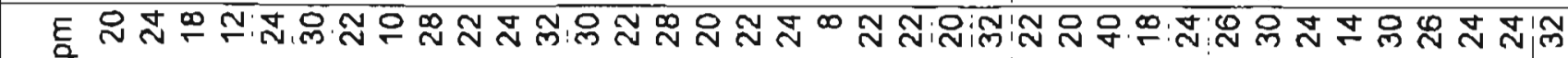
$\succ \frac{2}{2}$

능

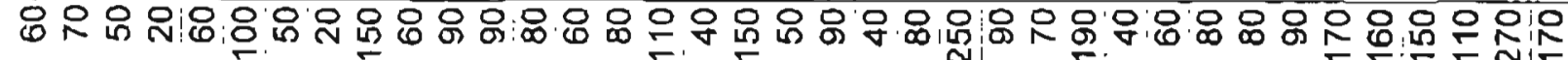

$\varepsilon$

노음

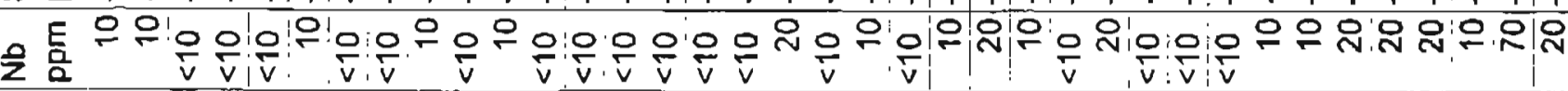

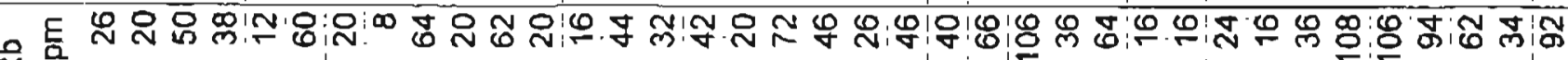
뜸

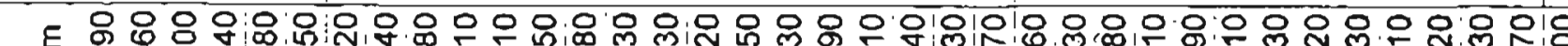
颉

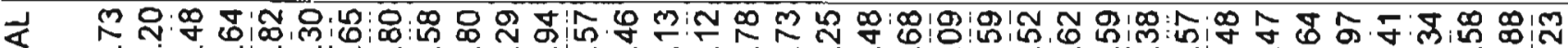
- I 은

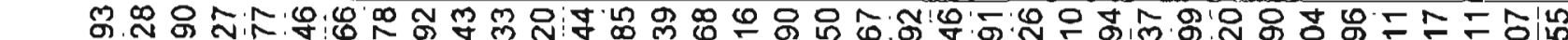

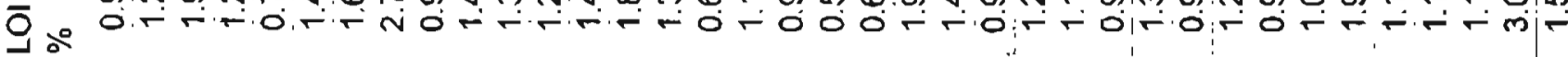

응

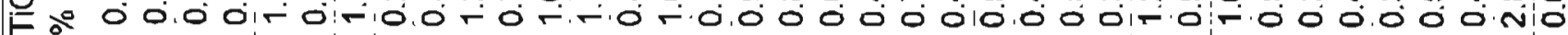

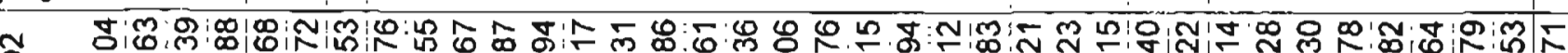

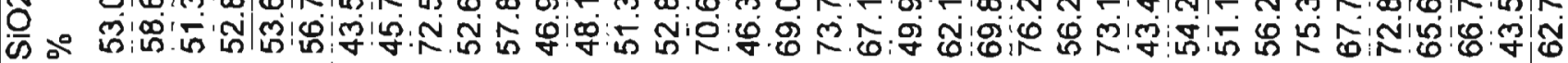

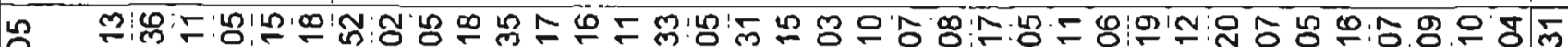

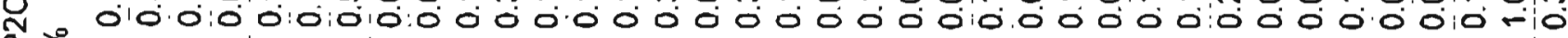
å

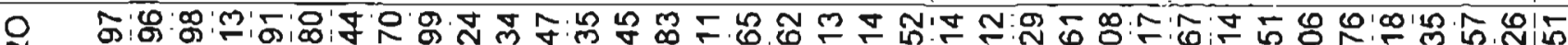

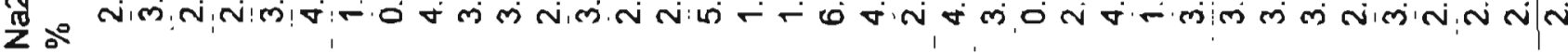

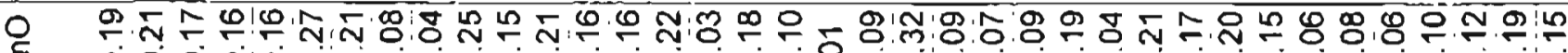

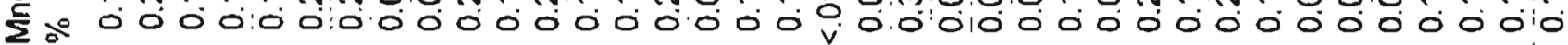

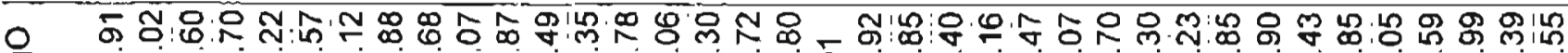
落

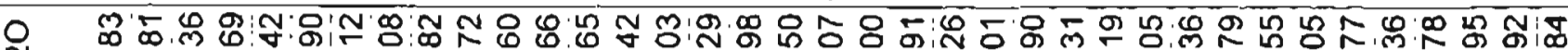

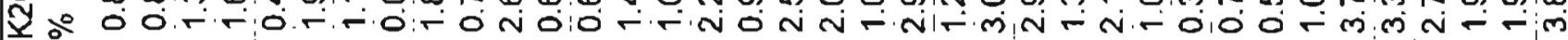

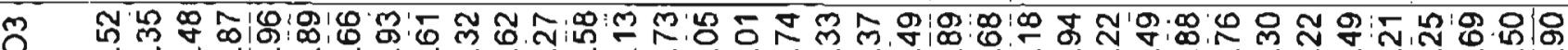

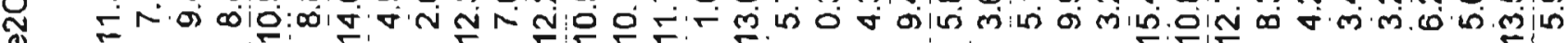

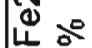

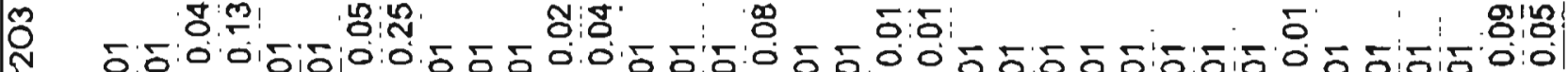

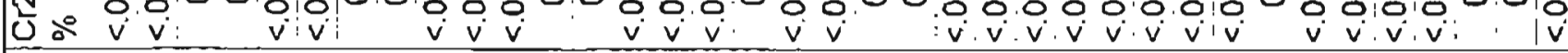

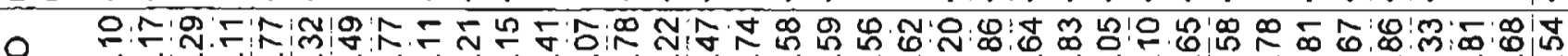

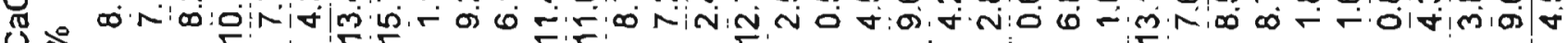

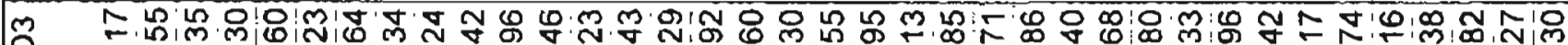

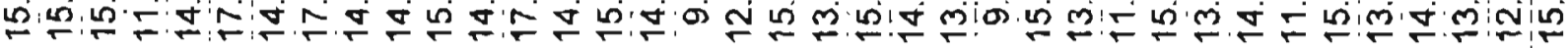
¿ㅇำ 
Table 4. Concentration of major-oxides, minor oxides, and trace elements in rock samples from the Eagle and Tanacross quadrangles, Alaska.

Note: -- = not analyzed.

\begin{tabular}{|c|c|c|c|c|c|c|c|c|c|c|c|c|c|c|c|c|c|c|c|c|}
\hline $\begin{array}{l}\text { SAMPLE } \\
\text { NUMBER }\end{array}$ & $\begin{array}{l}\mathrm{Al} 2 \mathrm{O} 3 \\
\% \\
\%\end{array}$ & $\begin{array}{l}\mathrm{CaO} \\
\% \ldots \\
\%\end{array}$ & $\begin{array}{l}\mathrm{Cr} 2 \mathrm{O} 3 \\
\%\end{array}$ & $\begin{array}{l}\mathrm{Fe} 2 \mathrm{O} 3 \\
\%\end{array}$ & $\mid \begin{array}{l}\mathrm{K} 2 \mathrm{O} \\
\% \\
\%\end{array}$ & $\begin{array}{l}\mathrm{MgO} \\
\%\end{array}$ & $\begin{array}{l}\mathrm{MnO} \\
\% \\
\%\end{array}$ & \begin{tabular}{|l}
$\mathrm{Na} 2 \mathrm{O}$ \\
$\overline{\%}-$
\end{tabular} & $\frac{\mathrm{P} 2 \mathrm{O} 5}{\%}$ & $\frac{\mathrm{SiO} 2}{\%}$ & $\mid \begin{array}{l}\mathrm{TiO} 2 \\
\% \\
\%\end{array}$ & LOI & \begin{tabular}{|l|} 
TOTAL \\
$\%$
\end{tabular} & Ba & $\begin{array}{l}\mathrm{Rb} \\
\mathrm{ppm}\end{array}$ & $\mid \begin{array}{l}\mathrm{Nb} \\
\mathrm{ppm}\end{array}$ & Sr & $\mid \begin{array}{l}Z \\
\text { pr } \\
\text { ppm }\end{array}$ & $\begin{array}{l}Y \\
\mathrm{ppm}\end{array}$ & $\begin{array}{c}\mathrm{BaO} \\
\%\end{array}$ \\
\hline $012158 \mathrm{E}$ & 13.56 & $3.5 \overline{8}$ & 0.01 & 4.97 & 2.48 & 2.39 & 0.09 & 2.13 & 0.08 & 67.90 & 0.42 & 1.94 & 99.70 & 1220 & 86 & 10 & |-- & 130 & 22 & 0.12 \\
\hline & 13.46 & 5.61 & 0.02 & 5.88 & 2.02 & 3.51 & 0.09 & 2.28 & 0.11 & 64.52 & 0.47 & 1.23 & 99.29 & 630 & 74 & 10 & -- & 110 & 24 & 0.06 \\
\hline $67 \mathrm{~B}$ & 15.96 & 1.34 & $<.01$ & 0.53 & 3.58 & 0.15 & 0.01 & 4.75 & 0.04 & 70.28 & 0.08 & 0.45 & 98.05 & 5240 & 72 & $<10$ & - & 40 & 8 & 0.78 \\
\hline $76 \bar{A}$ & 15.62 & 10.48 & 0.07 & 10.01 & 0.27 & 4.60 & 0.14 & 3.75 & 0.18 & 52.00 & 1.17 & 1.22 & 99.54 & 100 & 12 & 10 & - & 90 & 26 & $<.01$ \\
\hline $85 A$ & 16.58 & 6.89 & $<.01^{\circ}$ & $\overline{9} . \overline{3}$ & $0.8 \overline{2}$ & 5.43 & 0.16 & 4.72 & 0.09 & 51.50 & 0.97 & 1.77 & 98.50 & 310 & 28 & $<10^{-}$ & $=$ & 60 & 18 & 0.01 \\
\hline $\mathrm{Z} 186 \mathrm{~A}$ & 14.87 & 3.12 & $<.01$ & 3.43 & 0.55 & 0.77 & 0.06 & 4.37 & 0.10 & 71.24 & 0.35 & 0.62 & 99.52 & 160 & 18 & $<10$ & -- & 230 & 38 & $<.01$ \\
\hline $12209 \mathrm{~A}$ & 13.41 & 2.27 & $<.01$ & 3.66 & 1.78 & 0.79 & 0.07 & 3.78 & 0.07 & 70.38 & 0.38 & 1.58 & 98.30 & 840 & 54 & 10 & -- & 130 & 26 & 0.09 \\
\hline $012225 \mathrm{~A}$ & 9.62 & 0.52 & 0.01 & 2.45 & 3.56 & 0.69 & 0.04 & 1.52 & 0.07 & 78.78 & 0.38 & 1.23 & 99.00 & 1000 & 86 & 20 & - & 220 & 20 & 0.12 \\
\hline & 17.13 & 3.66 & $<01$ & 1.55 & 0.80 & 0.34 & 0.02 & 5.97 & 0.06 & 67.97 & 0.17 & 0.75 & 98.69 & 1000 & 20 & 10 & $=$ & 90 & 8 & 0.13 \\
\hline & 18.69 & 6.25 & $<.01$ & 10.54 & 1.00 & 3.11 & 0.16 & 4.82 & 0.11 & 52.52 & 0.84 & 1.08 & 99.26 & 510 & 30 & $<10$ & -- & 50 & 18 & 0.09 \\
\hline & 12.91 & 8.30 & 0.10 & 8.62 & 2.09 & $10.4 \hat{\imath}$ & 0.21 & 3.14 & 0.09 & 50.88 & 0.34 & 1.86 & 99.12 & 1250 & 44 & $<10$ & 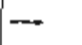 & 30 & 14 & 0.14 \\
\hline $5 \mathrm{~A}$ & 13.51 & $\overline{4} .75$ & $<.01$ & 10.78 & 0.35 & 3.17 & 0.24 & 3.41 & 0.18 & 59.62 & 0.98 & 1.14 & 98.17 & 230 & 16 & $<10$ & - & 80 & 30 & 0.01 \\
\hline & 14.30 & 5.08 & $<.01$ & 7.05 & 3.94 & 2.66 & 0.19 & 2.07 & 0.41 & 60.99 & 0.80 & 1.25 & 99.10 & 2330 & 82 & 20 & -- & 180 & 46 & 0.28 \\
\hline & 15.56 & 9.24 & $<.01$ & 12.32 & 1.21 & 3.80 & 0.18 & 3.38 & 0.38 & 51.03 & 1.50 & 0.69 & 99.34 & 240 & 24 & 10 & -- & 110 & 26 & 0.01 \\
\hline & 18.61 & 9.91 & $<.01$ & $8.6 \overline{6}$ & 1.12 & 4.35 & 0.14 & 3.87 & 0.08 & 50.12 & 0.68 & 1.59 & 99.19 & 310 & 32 & $<10^{\circ}$ & -- & 60 & 16 & 0.01 \\
\hline & 13.62 & 0.81 & $<.01$ & 3.23 & 4.76 & 0.79 & 0.08 & 3.55 & 0.05 & 70.55 & 0.31 & 0.44 & 98.38 & 1550 & 104 & 10 & - & 150 & 36 & 0.18 \\
\hline & 18.09 & 5.90 & $<.01$ & 6.94 & 3.13 & 2.42 & 0.06 & 3.04 & 0.19 & 56.28 & 0.95 & 1.19 & 98.36 & 1250 & 96 & $<10^{\circ}$ & - & 110 & 26 & 0.12 \\
\hline Z267A & 16.52 & 2.61 & $<.01$ & 2.73 & 3.28 & 1.17 & 0.05 & 4.25 & 0.08 & 67.31 & 0.35 & 0.72 & 99.40 & 2120 & 80 & 20 & $\cdots$ & 90 & 10 & 0.24 \\
\hline $1 \mathrm{Z272A}$ & 10.32 & 1.90 & $<.01$ & 2.84 & 1.26 & 0.74 & 0.05 & 3.02 & 0.09 & 76.48 & 0.40 & 1.52 & 98.69 & 630 & 54 & 20 & - & 240 & 24 & 0.06 \\
\hline
\end{tabular}


Table 4. Concentration of major-oxides, minor oxides, and trace elements in rock samples from the Eagle and Tanacross quadrangles, Alaska. Note: --- not analyzed.

\begin{tabular}{|c|c|c|c|c|c|c|c|}
\hline$\underset{\%}{\mathrm{SrO}}$ & $\begin{array}{l}\text { As } \\
\text { ppm }\end{array}$ & $\begin{array}{l}\text { Ce } \\
\text { ppm }\end{array}$ & $\begin{array}{l}\text { La } \\
\text { ppm }\end{array}$ & Th & ppm & $\begin{array}{l}\bar{W} \\
\overline{p p m}\end{array}$ & $\mid \begin{array}{l}\text { SAMPLE } \\
\text { NUMBER }\end{array}$ \\
\hline$\ldots$ & - & - & - & - & - & - & 99MBW079 \\
\hline - & - & - & - & - & - & -- & 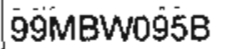 \\
\hline- & $-\infty$ & - & - & - & -- & - & OODS121 \\
\hline 0.03 & - & $=$ & - & - & $\therefore$ & - & $00 J G 014-6 / 28$ \\
\hline 0.03 & $=$ & $=$ & - & $=$ & 二- & - & $00 J 6032-6 / 29$ \\
\hline$<.01$ & - & - & 二 & - & -- & - & $00 J G 092-6 / 20$ \\
\hline 0.04 & - & - & - & - & - & - & 00JG095-6/20 \\
\hline$\ldots$ & - & - & - & - & - & - & OOMBW194 \\
\hline - & -- & -- & - & - & - & - & OOMBW213C \\
\hline- & -- & $\because$ & - & - & $-\ldots$ & - & OOMBW535 \\
\hline- & - & - & - & - & -+ & $=$ & OOMBW549 \\
\hline$\ldots$ & 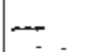 & --. & 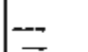 & 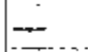 & $\ldots$ & - & OORFO01A \\
\hline$\ldots$ & -- & -- & $=$ & $\overline{-}$ & 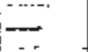 & $\overline{-r}$ & OORF054 \\
\hline-- & - & --- & - & - & $\therefore$ & $\because-$ & OORF 061 \\
\hline$\cdots$ & $\rightarrow$ & $-\cdots-$ & $-\infty$ & $-\infty$ & -- & --- & ODRFO61 \\
\hline- & --- & |-- & - & - & - & --- & OORF092A \\
\hline -- & $-\cdots$ & $-\cdots$ & $\cdots$ & $\cdots$ & - & - & DORF092A \\
\hline$\cdots$ & - & -- & 二 & - & - & - & OORF112A \\
\hline 二. & $=$ & $-\overline{-1}$ & $=$ & $\ldots$ & - & -- & OORF $113 \mathrm{~B}$ \\
\hline- & $=$ & 等- & $\overline{-}$ & - & $\cdots$ & $=$ & OORF 118 \\
\hline - & - & -- & -- & - & - & - & $O O R F 126 A$ \\
\hline - & - & - & - & - & $-\cdots$ & $\cdots$ & ODRF $126 \mathrm{~A}$ \\
\hline- & -- & --- & - & 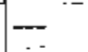 & $\ldots$ & $\cdots$ & OORF126B \\
\hline$\ldots$ & $\cdots$ & -- & - & $\frac{\cdots}{\cdots}$ & $\cdots$ & --- & OORF 134 \\
\hline- & $\cdots$ & $\therefore$ & $-\cdots$ & $\cdots$ & $\div$ & 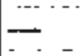 & OORF 134 \\
\hline- & -- & -- & - & $\cdots$ & - & - & OORF160 \\
\hline - & --- & - & - & - & $\cdots$ & $\ldots$ & OORF174A \\
\hline$\overline{-}$ & - & --- & - & - & - & - & DORF 201A \\
\hline$=$ & - & - & - & - & - & $\because$ & OORF $236 \mathrm{~A}$ \\
\hline - & - & --- & -- & $=$ & E- & $=$ & $00 R F 322 A$ \\
\hline$<01$ & - & 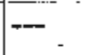 & -- & $\overline{-}$ & $=$ & $\because$ & $00 R F 340 B$ \\
\hline- & - & --- & -- & - & - & - & $00 \mathrm{RF} 345 \mathrm{~A}$ \\
\hline- & $\cdots$ & - & - & $\ldots$ & $=$ & $\because$ & OORF $349 \mathrm{~A}$ \\
\hline- & - & $=$ & - & - & $=$ & $\div$ & OORF $349 B$ \\
\hline$=$ & - & - & - & - & $=$ & $\because-$ & OORF $357 \mathrm{C}$ \\
\hline- & - & - & - & - & - & - & $0 \bar{Q} \overline{R F} \overline{37} \overline{6}$ \\
\hline -- & - & - & - & --- & - & - & 00RF 454 \\
\hline
\end{tabular}


Table 4. Concentration of major-oxides, minor oxides, and trace elements in rock samples from the Eagle and Tanacross quadrangles, Alaska. Note: $\ldots=$ not analyzed.

\begin{tabular}{|c|c|c|c|c|c|c|c|}
\hline $\begin{array}{c}\mathrm{SrO} \\
\%\end{array}$ & $\begin{array}{l}\text { As } \\
\text { ppm }\end{array}$ & $\begin{array}{l}\text { Ce } \\
\text { ppm }\end{array}$ & $\begin{array}{l}\text { La } \\
\text { ppm }\end{array}$ & $\begin{array}{l}\text { Th } \\
\text { ppm }\end{array}$ & $\begin{array}{l}U \\
\text { ppm }\end{array}$ & $\begin{array}{l}\text { W } \\
\text { ppm }\end{array}$ & $\begin{array}{l}\text { SAMPLE } \\
\text { NUMBER }\end{array}$ \\
\hline- & - & $\cdots$ & - & $=$ & --- & - & $00 R F 467$ \\
\hline- & $\ldots$ & - & - & -- & - & - & OORF 487B \\
\hline- & - & -- & - & -- & 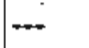 & --- & 00RF 488 \\
\hline- & $\ldots$ & --- & - & --- & - & - & OORF517 \\
\hline- & - & $=$ & - & $=$ & - & - & 00RF547B \\
\hline- & $\cdots$ & - & - & - & - & - & OORF $549 \mathrm{~A}$ \\
\hline- & - & - & - & - & - & - & OORF552 \\
\hline- & {$[--$} & - & $\therefore$ & - & 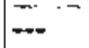 & - & $00 R F 563$ \\
\hline-- & - & - & -- & - & $\cdots$ & - & 00RF617 \\
\hline$\because$ & $\because$ & - & $\rightarrow$ & - & $=$ & - & OORF681 \\
\hline- & -- & - & $\therefore$ & - & $=$ & - & OORN204B \\
\hline- & -- & - & $\ldots$ & - & -- & - & OORN256A \\
\hline-- & - & - & - & - & -- & - & O0RN268A \\
\hline- & - & 一 & --- & -- & - & - & $00 \mathrm{RN} 277 \mathrm{~B}$ \\
\hline 0.01 & - & $\rightarrow$ & -- & - & --- & $\ldots$ & O0RN370A \\
\hline$\because$ & - & i- & - & $\rightarrow$ & - & $\ldots$ & OORN413 \\
\hline$\cdots$ & $\cdots$ & $\ldots$ & $\because-$ & -- & - & $\cdots$ & OORN454A \\
\hline$\rightarrow$ & - & - & - & $\div$ & -- & $=$ & OOWM117 \\
\hline$\cdots$ & - & - & - & $\because$ & - & - & OOWM225 \\
\hline 0.05 & $=-$ & $\cdots$ & --- & $-\cdots$ & - & - & $00 \mathrm{WM} 250 \mathrm{C}$ \\
\hline 0.03 & $-\infty$ & $=$ & - & - & - & $=$ & OOWM252B \\
\hline 0.01 & $=$ & 二 & - & - & 二 & $=$ & OOWM $321 \mathrm{~A}$ \\
\hline$\overline{0} . \overline{04}$ & $<5$ & 30 & 10 & $--\frac{4}{4}$ & $<4$ & $<10$ & $01 \mathrm{JEA} 2 \mathrm{~A}$ \\
\hline 0.02 & $<\overline{5}$ & 20 & 10 & 4 & $<4$ & $<10$ & 01 JEA35A \\
\hline 0.04 & $<5$ & 10 & $<10$ & $<4$ & $<4$ & $<10$ & 01JEA39B \\
\hline 0.03 & $<5$ & 20 & $<10$ & 4 & $<4$ & $<10$ & 01JEA47A \\
\hline 0.07 & 5 & 20 & 10 & $<4^{--}$ & $<4$ & $<10$ & O1JEA51B \\
\hline 0.11 & $\dot{5}$ & $<10$ & $<10$ & $<4$ & $<4$ & $<10$ & 01 JEA558 \\
\hline 0.05 & $<5$ & 20 & $<10$ & $<4$ & $<\dot{4}$ & $<10$ & 01JEA60A \\
\hline 0.05 & 5 & 20 & 10 & 4 & $<\overline{4}$ & $<10$ & 01.JEA90A \\
\hline$\overline{0} . \overline{0} \overline{9}$ & $\overline{5}$ & 70 & $40^{\circ}$ & $-1 \overline{2}$ & $<4$ & $<10$ & $01 \mathrm{JEA} 138 \mathrm{~A}$ \\
\hline 0.05 & $<5$ & 10 & $<10^{-}$ & $<4$ & $<4$ & $<10$ & $01 \mathrm{JEA} 238 \mathrm{~A}$ \\
\hline 0.03 & 5 & 40 & 20 & 12 & $<4$ & $<10$ & 01JEA243A \\
\hline 0.07 & 10 & 90 & 60 & 12 & $<4$ & $<10$ & 01JEA257B \\
\hline 0.03 & $<5$ & 30 & 10 & $<4$ & $<4$ & $<10$ & 01JEA259B \\
\hline 0.15 & 10 & $2 \overline{0}$ & $<10$ & 8 & $<4$ & $<10$ & 01JEA311B \\
\hline 0.15 & 10 & $<10$ & $<10$ & $\overline{4}$ & $<4$ & $<10$ & $01 \mathrm{KC} 8 \mathrm{D}$ \\
\hline
\end{tabular}


山

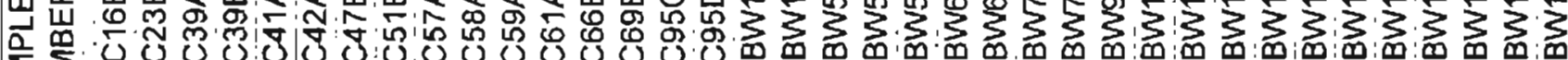

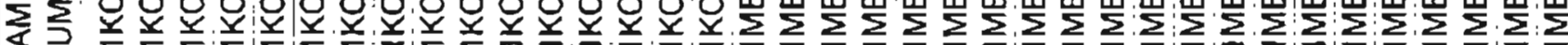

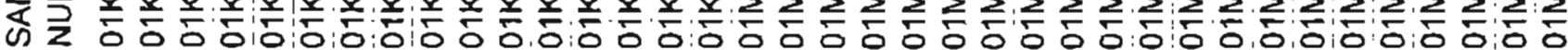

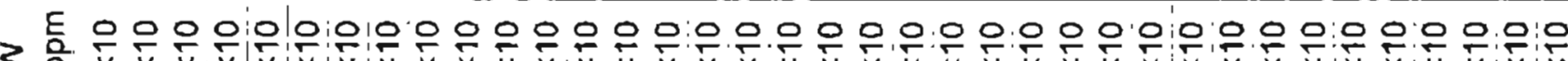
$3 \bar{a} \cdot \bar{v} \bar{v} \bar{v} \cdot \bar{v}|\bar{v}| \bar{v}|\bar{v}| \bar{v} \bar{v} \bar{v} \bar{v} \bar{v} \cdot \bar{v} \bar{v} \bar{v} \bar{v} \bar{v} \bar{v} \bar{v} \bar{v} \bar{v}_{\bar{v}} \bar{v} \bar{v} \bar{v} \bar{v} \bar{v} \bar{v}^{\prime} \bar{v} \bar{v} \bar{v} i \bar{v} \cdot \bar{v}: \bar{v} \bar{v} \cdot \bar{v} \mid \bar{v}$

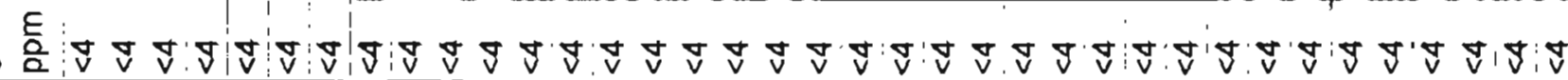

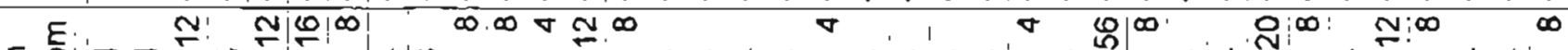

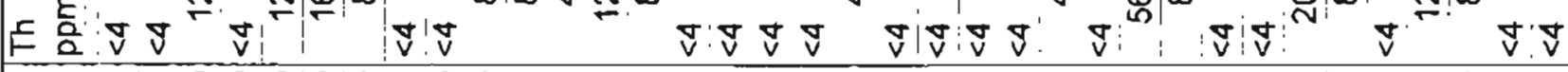

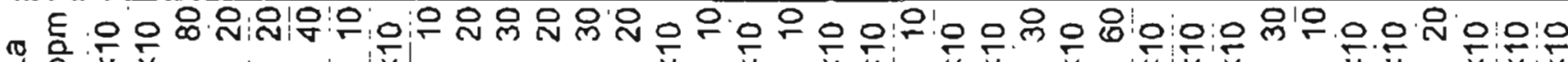

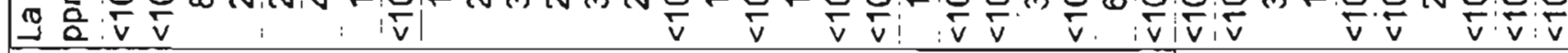

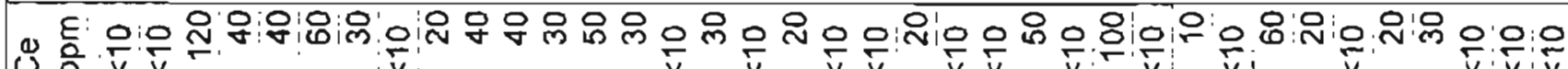

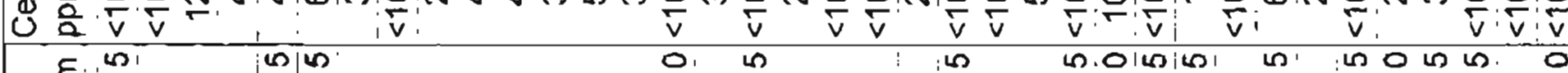

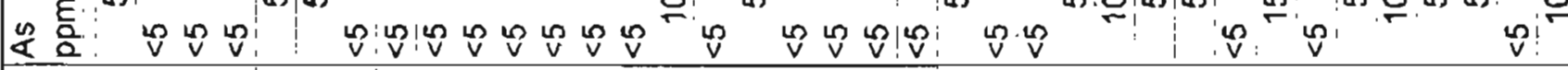

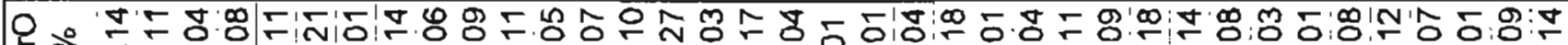

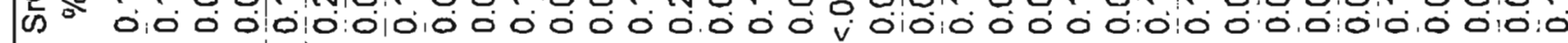


山出

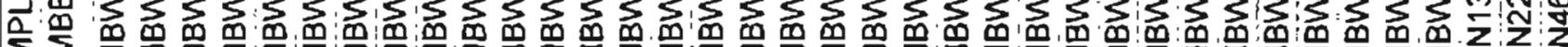

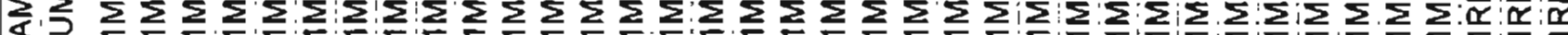
秐

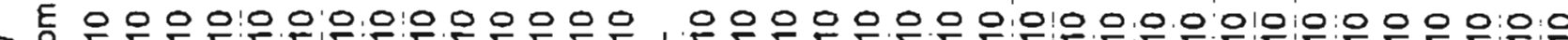
उ $\bar{\alpha} \cdot \bar{v} \bar{v} \bar{v} \bar{v} \cdot \bar{v} \bar{v}: \bar{v} i \bar{v}: \bar{v} \cdot \bar{v} \bar{v} \bar{v} \bar{v} \bar{v} \mid: \bar{v} \bar{v} \bar{v} \cdot \bar{v} \bar{v} \bar{v} \bar{v} \bar{v} i \bar{v}: \bar{v} i \bar{v} i \bar{v} i \bar{v} i \bar{v}: \bar{v} \bar{v}: \bar{v} \bar{v} \bar{v} \bar{v}: \bar{v}: \bar{v}$

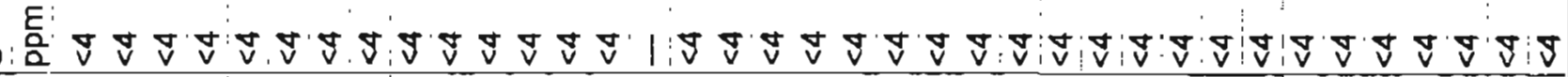

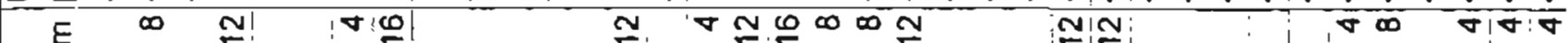

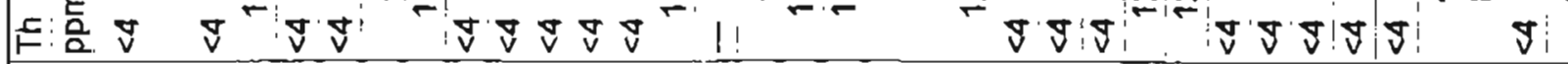

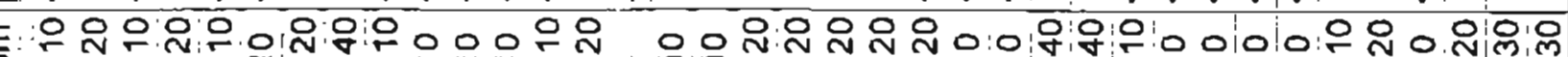
J 运 农告

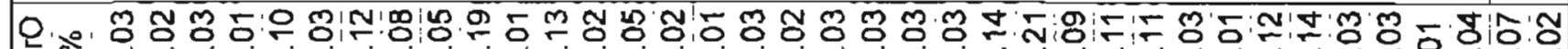

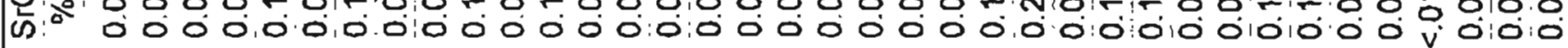


Table 4. Concentration of major-oxides, minor oxides, and trace elements in rock samples from the Eagle and Tanacross quadrangles, Alaska. Note: $\ldots=$ not analyzed.

\begin{tabular}{|c|c|c|c|c|c|c|c|}
\hline$\frac{\mathrm{SrO}}{\%}$ & $\begin{array}{l}\text { As } \\
\text { ppm }\end{array}$ & $\begin{array}{l}\text { Ce } \\
\text { ppm }\end{array}$ & $\begin{array}{l}\text { La } \\
\text { ppm }\end{array}$ & $\begin{array}{l}\text { Th } \\
\text { ppm }\end{array}$ & ppm & $\begin{array}{l}W \\
\mathrm{ppm}\end{array}$ & $\begin{array}{l}\text { SAMPLE } \\
\text { NUMBEER }\end{array}$ \\
\hline 0.02 & $<5$ & 10 & $<10$ & $<4$ & $<4$ & $<10$ & O1RN61B \\
\hline 0.05 & $<5$ & 60 & 40 & 8 & $<4$ & $<10$ & 01RN75A \\
\hline 0.04 & $<5$ & 10 & $<10$ & $<4$ & $<4$ & $<10$ & 01RN102B \\
\hline 0.01 & 20 & $<10^{\circ}$ & $<10$ & $<4$ & $<\dot{4}$ & $<10$ & 01RN $117 \mathrm{~A}$ \\
\hline 0.04 & $<5$ & 10 & $\leq 10$ & 4 & $<4$ & $<10$ & O1RN128A \\
\hline 0.05 & $<5$ & 30 & 10 & 4 & $<4$ & $<10$ & 01RN134B \\
\hline 0.09 & $<5$ & 20 & $<10$ & $<4$ & $<4$ & $<10$ & 01RN136A \\
\hline 0.01 & 35 & $<10$ & $<10$ & $<4$ & $<4$ & $<10$ & 01RN151A \\
\hline 0.01 & $<5$ & 50 & 30 & 8 & $<4$ & $<10$ & $O f R N 157 A$ \\
\hline 0.04 & $<5$ & $10^{\circ}$ & $<10^{\circ}$ & $<4^{\circ}$ & $<4$ & $<10$ & 01RN167A \\
\hline 0.12 & $<5$ & 40 & 20 & 8 & $<\dot{4}$ & $<10$ & 01RN171A \\
\hline 0.03 & $<5$ & 10 & $<10^{-}$ & $<4$ & $<4$ & $<10$ & $01211 \mathrm{~A}$ \\
\hline 0.04 & $<5$ & 10 & $<10$ & $<4$ & $<4$ & $<10$ & 01Z19A \\
\hline 0.02 & $<5$ & 10 & $<10$ & $<4$ & $<4$ & $<10$ & $01221 \mathrm{~A}$ \\
\hline 0.04 & $<5$ & 30 & 20 & $<4$ & $<4$ & $<10$ & $01233 \mathrm{~B}$ \\
\hline 0.18 & 5 & 70 & 30 & 8 & $<4$ & $<10$ & 01235A \\
\hline 0.02 & $<5$ & 20 & $<10$ & $<4$ & $<4$ & $<10$ & $01236 \mathrm{~A}$ \\
\hline 0.02 & 5 & 50 & 20 & 8 & $<4$ & $<10$ & $01250 \mathrm{~A}$ \\
\hline 0.09 & 5 & $<10$ & $<10$ & $<4$ & $<4$ & $<10$ & $01258 \mathrm{~A}$ \\
\hline 0.03 & $\leq 5$ & 30 & 10 & $\ldots 8$ & $<4$. & $<10$ & 012538 \\
\hline 0.03 & $<5$ & $<10$ & $<10$ & $<4$ & $<4$ & $<10$ & 01261A \\
\hline 0.03 & $<5$ & 10 & $<10$ & $<4$ & $<4$ & $<10$ & 01Z63B \\
\hline 0.14 & 5 & 90 & 60 & 24 & $<4$ & $<10$ & $03265 \mathrm{~A}$ \\
\hline 0.01 & 10 & 50 & 20 & 12 & $<4$ & $<10$ & $01270 \mathrm{~A}$ \\
\hline 0.05 & $<\underline{5}$ & 20 & 10 & $\leq 4$ & $<4$ & $\leq 10$ & $01 Z 72 \mathrm{~A}$ \\
\hline 0.03 & $<5$ & 50 & 30 & 12 & $<4$ & $<10$ & 01Z81A \\
\hline 0.03 & $<5$ & 10 & $<10$ & $<4$ & $<4$ & $<10$ & 01Z82A \\
\hline 0.03 & $<5$ & 10 & $<10$ & $<4$ & $\leq 4$ & $\leq 10$ & $01 Z 92 \mathrm{~A}$ \\
\hline 0.03 & $<5$ & 20 & $<10$ & 4 & $<4$ & $<10$ & $01293 \mathrm{~A}$ \\
\hline 0.02 & $<5$ & 20 & 10 & $<4$ & $<4$ & $<10$ & 01Z95A \\
\hline 0.01 & $<5$ & 20 & 10 & $<4$ & $<4$ & $<10$ & $01 Z 98 A$ \\
\hline 0.07 & 5 & 30 & 10 & 8 & $<4$ & $<10$ & $01 Z 108 \mathrm{~A}$ \\
\hline 0.01 & $<5$ & 50 & 20 & 12 & $<4$ & $<10$ & $01 \mathrm{Z} 121 \mathrm{~A}$ \\
\hline 0.02 & $<5$ & 40 & 20 & 12 & $<4$ & $<10$ & $01 Z 126 \mathrm{~A}$ \\
\hline 0.02 & $<5$ & 40 & 20 & 8 & $<4$ & $<10$ & $01 Z 132 A$ \\
\hline 0.11 & 10 & 100 & 60 & 12 & $<4$ & $<10$ & $01 Z 158 \mathrm{~A}$ \\
\hline 0.09 & $<5$ & 90 & 50 & 16 & $<4$ & $<10$ & $012158 \mathrm{C}$ \\
\hline
\end{tabular}


Table 4. Concentration of major-oxides, minor oxides, and trace elements in rock samples from the Eagle and Tanacross quadrangles, Alaska. Note: $\ldots .=$ not analyzed.

\begin{tabular}{|c|c|c|c|c|c|c|c|}
\hline $\begin{array}{c}\mathrm{SrO} \\
\%\end{array}$ & $\begin{array}{l}\text { As } \\
\text { ppm }\end{array}$ & $\begin{array}{l}\text { Ce } \\
\text { ppm }\end{array}$ & La & $\begin{array}{l}\text { Th } \\
\text { ppm }\end{array}$ & $\begin{array}{l}\mathrm{u} \\
\mathrm{ppm}\end{array}$ & $\begin{array}{l}W \\
\mathrm{ppm}\end{array}$ & $\begin{array}{l}\text { SAMPLE } \\
\text { NUMBER }\end{array}$ \\
\hline $0 . \overline{0} 3$ & $<5$ & 40 & 20 & 12 & $<4$ & $<10$ & $01 Z 158 \mathrm{E}$ \\
\hline 0.03 & 5 & 40 & 20 & 12 & $<\overline{4}$ & $<10$ & $012160 \mathrm{~A}$ \\
\hline 0.10 & $<5^{--}$ & $<10^{-}$ & $<10$ & $<4$ & $<4$ & $<10$ & $012167 \mathrm{~B}$ \\
\hline 0.03 & $<5$ & 20 & 10 & $<4$ & $<4$ & $<10$ & $012176 \mathrm{~A}$ \\
\hline 0.03 & $<5$ & 10 & $<10$ & $<4$ & $<4$ & $<10$ & $01 Z 185 \mathrm{~A}$ \\
\hline 0.04 & $<5$ & 40 & 20 & 8 & $<4$ & $<10^{\circ}$ & $012186 \mathrm{~A}$ \\
\hline 0.04 & $<\overline{5}$ & 40 & $\overline{2} \overline{0}$ & $1 \ddot{2}$ & $<4$ & $<10$ & $01 \overline{2} 209 \mathrm{~A}$ \\
\hline 0.01 & 10 & $4 \overline{0}$ & 20 & -16 & $<\ddot{4}$ & $<10$ & $01 Z 225 \dot{A}$ \\
\hline 0.14 & $<5$ & $<10$ & $<10$ & $<4$ & $<4$ & $<10$ & $04 Z 230 \mathrm{~A}$ \\
\hline 0.05 & $<5$ & $<10$ & $<10$ & $<4$ & $<4$ & $<10$ & $01 \bar{Z} 235 \mathrm{~A}$ \\
\hline 0.03 & 20 & $<10$ & $<10$ & $<4$ & $<4$ & $<10$ & $012237 \mathrm{~A}$ \\
\hline 0.03 & $<5$ & 20 & $<10$ & $<4$ & $<4$ & $<10^{\circ}$ & $01 \mathrm{Z} 245 \mathrm{~A}$ \\
\hline$\overline{0} . \overline{0}$ & 5 & 80 & 50 & 16 & $<4$ & $<\overline{10}$ & $012250 \mathrm{~A}$ \\
\hline 0.04 & $<5^{\circ}$ & 30 & jo & $<4^{--}$ & $<4$ & $<10$ & $01 Z 254 A$ \\
\hline 0.05 & $<5$ & 10 & $<10^{-}$ & $<4$ & $<4$ & $<10$ & $01225 \overline{6}$ \\
\hline 0.01 & $<5$ & 30 & 10 & 4 & $<4$ & $<10$ & $01 \mathrm{Z} 258 \mathrm{~A}$ \\
\hline 0.05 & $<5$ & 30 & 10 & 4 & $<4$ & $<10$ & $01 Z 262 \mathrm{~A}$ \\
\hline 0.09 & $<5$ & 10 & $<10$ & $<4$ & $<4$ & $<10$ & $01 Z 267 \mathrm{~A}$ \\
\hline 0.01 & 10 & $60^{\circ}$ & $\because 40$ & ${ }^{\cdots} 16$ & $<4$ & $<10$ & $01 Z 272 A$ \\
\hline
\end{tabular}


Table 5. Concentration of rare-earth elements in rock samples collected in the Eagle and Tanacross quadrangles.

\begin{tabular}{|c|c|c|c|c|c|c|c|c|c|c|c|c|c|c|c|c|c|}
\hline $\begin{array}{l}\text { SAMPLE } \\
\text { DESCRIPTION }\end{array}$ & $\begin{array}{l}\text { Ce } \\
\text { ppm }\end{array}$ & $\begin{array}{l}\text { Dy } \\
\text { ppm }\end{array}$ & $\begin{array}{l}\text { Er } \\
\text { ppm }\end{array}$ & $\begin{array}{l}\text { Eu } \\
\text { ppm }\end{array}$ & $\begin{array}{l}\text { Gd } \\
\text { ppm }\end{array}$ & $\begin{array}{l}\text { Ho } \\
\text { ppm }\end{array}$ & $\begin{array}{l}\text { La } \\
\text { ppm }\end{array}$ & $\begin{array}{l}\text { Lu } \\
\text { ppm }\end{array}$ & $\begin{array}{l}\mathrm{Nd} \\
\mathrm{ppm}\end{array}$ & $\begin{array}{l}\mathrm{Pr} \\
\mathrm{Ppm}\end{array}$ & $\begin{array}{l}\text { Sm } \\
\text { ppm }\end{array}$ & $\begin{array}{l}\text { Tb } \\
\mathrm{ppm}\end{array}$ & $\begin{array}{l}\text { Th } \\
\text { ppm }\end{array}$ & $\begin{array}{l}\mathrm{Tm} \\
\mathrm{ppm}\end{array}$ & U & $\begin{array}{l}Y \\
\text { ppm }\end{array}$ & $\begin{array}{l}\text { Yb } \\
\text { ppm }\end{array}$ \\
\hline OODS020 & 15 & 4.1 & 3.2 & 1 & 3.4 & 1 & 6 & 0.4 & 10 & 2.2 & 2.7 & 0.6 & $<1$ & 0.4 & 0.5 & 25.5 & 2.5 \\
\hline OORN143 & 15.5 & $2 . \dot{5}$ & 1.8 & 0.8 & 2.4 & 0.6 & 6.5 & 0.2 & 10 & 2.3 & 2.6 & 0.4 & $<1$ & 0.2 & 0.5 & 15.5 & 1.7 \\
\hline $01 \mathrm{JEA} 238 \mathrm{~A}$ & 13.5 & 2.8 & 1.7 & 0.7 & 2.5 & 0.6 & 6 & 0.3 & 7.5 & 1.7 & 1.9 & 0.4 & $<1$ & 0.2 & $<.5$ & 16 & 1.7 \\
\hline $01 \mathrm{MBW} 187 \mathrm{~A}$ & 23.5 & $\overline{7} . \overline{1}$ & 4.3 & 1.6 & 6.2 & 1.5 & 9.5 & 0.7 & 16.5 & 3.3 & $4 . \overline{7}$ & 1 & 1 & 0.6 & $<.5$ & 40 & 4.2 \\
\hline $01 \mathrm{MBW} 287 \mathrm{~B}$ & $-1 \overline{6}$ & $\overline{2.9}$ & $\bar{\jmath} . \overline{7}$ & $0 . \overline{7}$ & 2.8 & $0 . \ddot{6}$ & $\overline{9}$ & $0 . \overline{3}$ & $\cdots \overline{9}$ & $2 . \overline{2}$ & 2.2 & 0.5 & 1 & 0.2 & 0.5 & 18 & 1.8 \\
\hline $01 \mathrm{MBW} 304 \mathrm{~A}$ & 20 & 6 & 3.7 & 1.5 & 5.5 & 1.2 & 8 & 0.6 & 14 & $2 . \overline{8}$ & 4.2 & 0.9 & $\leq 1$ & 0.5 & $<.5^{-}$ & 33.5 & 3.3 \\
\hline $01 \mathrm{RN} 10 \overline{2} \mathrm{~B}$ & 10.5 & 2.2 & $\overline{1} . \overline{4}$ & $0 . \overline{7}$ & 2 & 0.5 & 5 & 0.2 & $\overline{6}$ & 1.3 & $1 . \overline{7}$ & 0.3 & $<1$ & $0 . \overline{1}$ & $<. \overline{5}$ & -13 & 1.4 \\
\hline 01RN167A & 14 & 3.5 & 2.1 & 1 & 3.3 & 0.7 & 5.5 & 0.3 & 9.5 & 1.9 & 2.6 & 0.5 & $<1$ & 0.3 & $<.5$ & 19.5 & 2 \\
\hline $01 Z 092 A$ & 14.5 & 3.6 & 2.4 & 1 & 3.2 & 0.7 & 6.5 & 0.3 & 8.5 & 1.9 & 2.4 & 0.6 & $<1$ & 0.3 & $<.5$ & 21 & 2.4 \\
\hline $012185 \mathrm{~A}$ & 12.5 & 3.1 & 1.9 & 0.8 & 2.7 & 0.6 & 5 & 0.3 & 8 & 1.7 & 2.2 & 0.5 & $<1$ & 0.3 & $<.5$ & 17.5 & 1.8 \\
\hline SAMPLE & UTM E & & UTMN & & \multicolumn{3}{|c|}{ Quadrangle } & \multicolumn{9}{|c|}{ Rock Type } & \\
\hline $000 \$ 020$ & 453364 & & 7119650 & & \multirow{2}{*}{\multicolumn{2}{|c|}{$\begin{array}{l}\text { Eagle A-2 } \\
\text { Eagle A-2 }\end{array}$}} & & \multirow{2}{*}{\multicolumn{5}{|c|}{$\begin{array}{l}\text { Biotite-rich amphibolite } \\
\text { Honblende plagioclase }\end{array}$}} & & & & & \\
\hline OORN143 & 465687 & & 7118146 & & & & & & & & & & & amp & Hibolit & & \\
\hline
\end{tabular}


Table 6. Coal Quality Analysis of Coal-bearing Tertiary Sedimentary Rocks in the Chicken Area.

\begin{tabular}{|c|c|c|c|c|c|}
\hline Coal Analysis & $\therefore$ & Geochemica! Testing, & division of Ene & Center & Inc. \\
\hline $\begin{array}{l}\text { Chicken Creek } \\
\text { Sample \# }\end{array}$ & $\begin{array}{l}\text { UTM E } \\
454630\end{array}$ & $\begin{array}{c}\text { UTM N } \\
7105798\end{array}$ & $\begin{array}{c}\text { Quadrangie } \\
\text { Eagle A-2 }\end{array}$ & & \\
\hline & $\begin{array}{c}\text { Air Dry Loss (\%) } \\
19.91\end{array}$ & $\begin{array}{c}\text { Residual Moisture (\%) } \\
9.02\end{array}$ & As Received & Dry & Dry Ash-Free \\
\hline Proximate Analysis & & & & & \\
\hline & $\begin{array}{l}\text { Moisture } \\
\text { Ash }\end{array}$ & & $\begin{array}{l}27.13 \\
23.80\end{array}$ & 3267 & 5193 \\
\hline & Volatile Matter & & 25.48 & 34.96 & 48.07 \\
\hline & Fixed Carbon & & 23.59 & 32.37 & \\
\hline Ultimate Analysis & & & & & \\
\hline & $\begin{array}{l}\text { Hydrogen } \\
\text { Carbon }\end{array}$ & & $\begin{array}{c}5.21 \\
32.77\end{array}$ & $\begin{array}{l}2.98 \\
44.97\end{array}$ & $\begin{array}{c}4.42 \\
66.78\end{array}$ \\
\hline & Nitrogen & & 0.66 & 0.90 & 1.34 \\
\hline & Sulfur & & 0.23 & 0.32 & 0.47 \\
\hline & Oxygen & & 37.33 & 18.16 & 26.99 \\
\hline & Ash & & 23.80 & 32.67 & \\
\hline $\begin{array}{l}\text { Heating Value (BTU/Lb) } \\
\text { Free Swelling Index } \\
\text { Equilibrim Moisture }\end{array}$ & . & & $\begin{array}{c}5,310 \\
0.5 \\
26.71\end{array}$ & 7,287 & $\{0,823$ \\
\hline
\end{tabular}


Table 7. Detection limits for geochemical analyses.

(Analytical methods: FA-AAS = Fire Assay-Atomic Absorption Spectroscopy, ICP-AES = Inductively Coupled Plasma-Atornic Emission Spectroscopy, FA-GRAV = Fire AssayGravimetric. FA-SCP-ARRAY = Fire Assay-Inductively Coupled Plasma, FA-ICPMS = Fire Assay-Inductively Coupled Plasma Mass Specurometry and AAS = Atomic Emission Spectroscopy) (ppm=parts per million. ppbripans per billion. \%=percent, $g / r=g r a n s / t o n n e)$

\begin{tabular}{|l|c|c|c|c|}
\hline Element & Units & $\begin{array}{c}\text { Lower } \\
\text { Detection } \\
\text { Limit }\end{array}$ & $\begin{array}{c}\text { Upper } \\
\text { Detection } \\
\text { Limit }\end{array}$ & $\begin{array}{c}\text { Analytical } \\
\text { Method }\end{array}$ \\
\hline $\mathrm{Au}$ & $\mathrm{ppb}$ & 5 & 10,000 & FA-AAS \\
\hline $\mathrm{Ag}$ & $\mathrm{ppm}$ & 0.2 & 100 & ICP-AES \\
\hline $\mathrm{Al}$ & percent & 0.01 & 15 & ICP-AES \\
\hline $\mathrm{As}$ & $\mathrm{ppm}$ & 2 & 10,000 & ICP-AES \\
\hline $\mathrm{B}$ & $\mathrm{ppm}$ & 10 & 10,000 & ICP-AES \\
\hline $\mathrm{Ba}$ & $\mathrm{ppm}$ & 10 & 10,000 & ICP-AES \\
\hline $\mathrm{Be}$ & $\mathrm{ppm}$ & 0.5 & 100 & ICP-AES \\
\hline $\mathrm{Bi}$ & $\mathrm{ppm}$ & 2 & 10,000 & ICP-AES \\
\hline $\mathrm{Ca}$ & percent & 0.01 & 15 & ICP-AES \\
\hline $\mathrm{Cd}$ & $\mathrm{ppm}$ & 0.5 & 500 & ICP-AES \\
\hline $\mathrm{Co}$ & $\mathrm{ppm}$ & 1 & 10,000 & ICP-AES \\
\hline $\mathrm{Cr}$ & $\mathrm{ppm}$ & 1 & 10,000 & ICP-AES \\
\hline $\mathrm{Cu}$ & $\mathrm{ppm}$ & 1 & 10,000 & ICP-AES \\
\hline $\mathrm{Fe}$ & $\mathrm{percent}$ & 0.01 & 15 & ICP-AES \\
\hline $\mathrm{Ga}$ & $\mathrm{ppm}$ & 10 & 10,000 & ICP-AES \\
\hline $\mathrm{Hg}$ & $\mathrm{ppm}$ & 1 & 10,000 & ICP-AES \\
\hline $\mathrm{K}$ & percent & 0.01 & 10 & ICP-AES \\
\hline $\mathrm{La}$ & $\mathrm{ppm}$ & 10 & 10,000 & ICP-AES \\
\hline $\mathrm{Mg}$ & percent & 0.01 & 15 & ICP-AES \\
\hline $\mathrm{Mn}$ & $\mathrm{ppm}$ & 5 & 10,000 & ICP-AES \\
\hline $\mathrm{Mo}$ & $\mathrm{ppm}$ & 1 & 10,000 & ICP-AES \\
\hline $\mathrm{Na}$ & percent & 0.01 & 10 & ICP-AES \\
\hline $\mathrm{Ni}$ & $\mathrm{ppm}$ & 1 & 10,000 & ICP-AES \\
\hline $\mathrm{P}$ & $\mathrm{ppm}$ & 10 & 10,000 & ICP-AES \\
\hline $\mathrm{Pb}$ & $\mathrm{ppm}$ & 2 & 10,000 & ICP-AES \\
\hline $\mathrm{S}$ & $\mathrm{percent}$ & 0.01 & 10 & ICP-AES \\
\hline $\mathrm{Sb}$ & $\mathrm{ppm}$ & 2 & 10,000 & ICP-AES \\
\hline $\mathrm{Sc}$ & $\mathrm{ppm}$ & 1 & 10,000 & ICP-AES \\
\hline $\mathrm{Sr}$ & $\mathrm{ppm}$ & 1 & 10,000 & ICP-AES \\
\hline $\mathrm{Ti}$ & $\mathrm{percent}$ & 0.01 & 10 & ICP-AES \\
\hline $\mathrm{T}$ & $\mathrm{ppm}$ & 10 & 10,000 & ICP-AES \\
\hline $\mathrm{U}$ & $\mathrm{ppm}$ & 10 & 10,000 & ICP-AES \\
\hline $\mathrm{V}$ & $\mathrm{ppm}$ & 1 & 10,000 & ICP-AES \\
\hline $\mathrm{W}$ & $\mathrm{ppm}$ & 10 & 10,000 & ICP-AES \\
\hline $\mathrm{Zn}$ & $\mathrm{ppm}$ & 2 & 10,000 & ICP-AES \\
\hline $\mathrm{Au} *$ & $\mathrm{ppb}$ & 1 & 1000 & FA-ICPMS \\
\hline $\mathrm{Pt}$ & $\mathrm{ppb}$ & 1 & 1000 & FA-ICPMS \\
\hline $\mathrm{Pd}$ & & & & FA-ICPMS \\
\hline & & 100 & \\
\hline
\end{tabular}


Table 8. Detection limits for major-oxide, minor-oxide and trace, and rare-eanth element analyses. ( $(\mathrm{LOI}=$ Loss on ignition. ppm $=$ parts per million. $\mathrm{XRF}=\mathrm{X}$-ray diffraction)

\begin{tabular}{|c|c|c|c|c|}
\hline Element & Units & $\begin{array}{c}\text { Lower } \\
\text { Detection } \\
\text { Linit }\end{array}$ & $\begin{array}{c}\text { Upper } \\
\text { Detection } \\
\text { Limit }\end{array}$ & $\begin{array}{c}\text { Analytical } \\
\text { Method }\end{array}$ \\
\hline $\mathrm{Al}_{2} \mathrm{O}_{3}$ & percent & 0.01 & 100.00 & XRF \\
\hline $\mathrm{BaO}$ & percent & 0.01 & 100.00 & $\mathrm{XRF}$ \\
\hline $\mathrm{CaO}$ & percent & 0.01 & 100.00 & XRF \\
\hline $\mathrm{Cr}_{2} \mathrm{O}_{3}$ & percent & 0.01 & 100.00 & $\mathrm{XRF}$ \\
\hline $\mathrm{Fe}_{2} \mathrm{O}_{3}$ & percent & 0.01 & 100.00 & $\mathrm{XRF}$ \\
\hline $\mathrm{K}_{2} \mathrm{O}$ & percent & 0.01 & 100.00 & XRF \\
\hline $\mathrm{MgO}$ & percent & 0.01 & 100.00 & $\mathrm{XRF}$ \\
\hline $\mathrm{MnO}$ & percent & 0.01 & 100.00 & XRF \\
\hline $\mathrm{Na}_{2} \mathrm{O}$ & percent & 0.01 & 100.00 & $\mathrm{XRF}$ \\
\hline $\mathrm{P}_{2} \mathrm{O}_{5}$ & percent & 0.01 & 100.00 & $\mathrm{XRF}$ \\
\hline $\mathrm{SiO}_{2}$ & percent & 0.01 & 100.00 & $\mathrm{XRF}$ \\
\hline SrO & percent & 0.01 & 100.00 & $\mathrm{XRF}$ \\
\hline $\mathrm{TiO}_{2}$ & percent & 0.01 & 100.00 & $\mathrm{XRF}$ \\
\hline $\mathrm{LOI}^{*}$ & percent & 0.01 & 100.00 & XRF \\
\hline Total & percent & 0.01 & 105.00 & Calculation \\
\hline As & epm & 5 & 5000 & XRF \\
\hline $\mathrm{Ba}$ & ppm & 10 & 10,000 & XRF \\
\hline $\mathrm{Ce}$ & ppm & 10 & 10.000 & $\mathrm{XRF}$ \\
\hline $\mathrm{La}$ & $\mathrm{ppm}$ & 10 & 10.000 & $\mathrm{XRF}$ \\
\hline $\mathrm{Nb}$ & $\mathrm{Ppm}$ & 10 & 10,000 & $X R F$ \\
\hline $\mathrm{Rb}$ & pom & 2 & 10,000 & $\mathrm{XRF}$ \\
\hline $\mathrm{Sr}$ & $\mathrm{pgm}$ & 2 & 10.000 & XRF \\
\hline Th & $\mathrm{ppm}$ & 4 & 10.000 & $\mathrm{XRF}$ \\
\hline $\mathrm{U}$ & $\mathrm{ppm}$ & 4 & 10,000 & XRF \\
\hline $\mathrm{W}$ & ppm & 10 & 10.000 & $\mathrm{XRF}$ \\
\hline$Y$ & $\mathrm{ppm}$ & 2 & 10,000 & XRF \\
\hline $\mathrm{Zr}$ & $\mathrm{ppm}$ & 10 & 10.000 & XRF \\
\hline
\end{tabular}


Table 9. Detection limits for rare-earth element analyses in Table 5.

(ppm = parts per million, ICP-MS = Inductively Coupled Plasma Mars Spectromery)

\begin{tabular}{|l|c|c|c|c|}
\hline Element & Units & $\begin{array}{c}\text { Lower } \\
\text { Detection } \\
\text { Limit }\end{array}$ & $\begin{array}{c}\text { Upper } \\
\text { Detection } \\
\text { Limit }\end{array}$ & $\begin{array}{c}\text { Analytical } \\
\text { Method }\end{array}$ \\
\hline Ce & ppm & 0.5 & 10,000 & ICP-MS \\
\hline $\mathrm{Dy}$ & $\mathrm{ppm}$ & 0.1 & 1,000 & ICP-MS \\
\hline Er & $\mathrm{ppm}$ & 0.1 & 1,000 & ICP-MS \\
\hline Eu & $\mathrm{ppm}$ & 0.1 & 1,000 & ICP-MS \\
\hline $\mathrm{Gd}$ & $\mathrm{ppm}$ & 0.1 & 1,000 & ICP-MS \\
\hline $\mathrm{Ho}$ & $\mathrm{ppm}$ & 0.1 & 1,000 & ICP-MS \\
\hline $\mathrm{La}$ & $\mathrm{ppm}$ & 0.5 & 10,000 & ICP-MS \\
\hline $\mathrm{Lu}$ & $\mathrm{ppm}$ & 0.1 & 1,000 & ICP-MS \\
\hline $\mathrm{Nd}$ & $\mathrm{ppm}$ & 0.5 & 10,000 & ICP-MS \\
\hline $\mathrm{Pr}$ & $\mathrm{ppm}$ & 0.1 & 1,000 & ICP-MS \\
\hline $\mathrm{Sm}$ & $\mathrm{ppm}$ & 0.1 & 1,000 & ICP-MS \\
\hline $\mathrm{Tb}$ & $\mathrm{ppm}$ & 0.1 & 1,000 & ICP-MS \\
\hline $\mathrm{Th}$ & $\mathrm{ppm}$ & 1 & 1,000 & ICP-MS \\
\hline $\mathrm{Tm}$ & $\mathrm{ppm}$ & 0.1 & 1,000 & ICP-MS \\
\hline $\mathrm{U}$ & $\mathrm{ppm}$ & 0.5 & 1,000 & ICP-MS \\
\hline $\mathrm{Y}$ & $\mathrm{ppm}$ & 0.5 & 1,000 & ICP-MS \\
\hline $\mathrm{Yb}$ & $\mathrm{ppm}$ & 0.1 & 1,000 & ICP-MS \\
\hline
\end{tabular}

